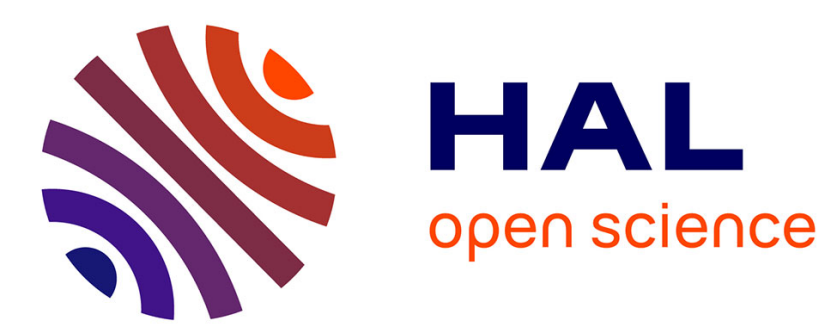

\title{
On Feedback Resource Allocation in Multiple-Input-Single-Output Systems Using Partial CSI Feedback
}

Behrooz Makki, Tommy Svensson, Thomas Eriksson, Mérouane Debbah

\section{- To cite this version:}

Behrooz Makki, Tommy Svensson, Thomas Eriksson, Mérouane Debbah. On Feedback Resource Allocation in Multiple-Input-Single-Output Systems Using Partial CSI Feedback. IEEE Transactions on Communications, 2015, 63 (3), pp.816 - 825. 10.1109/TCOMM.2015.2398861 . hal-01241743

\author{
HAL Id: hal-01241743 \\ https://hal.science/hal-01241743
}

Submitted on 10 Dec 2015

HAL is a multi-disciplinary open access archive for the deposit and dissemination of scientific research documents, whether they are published or not. The documents may come from teaching and research institutions in France or abroad, or from public or private research centers.
L'archive ouverte pluridisciplinaire HAL, est destinée au dépôt et à la diffusion de documents scientifiques de niveau recherche, publiés ou non, émanant des établissements d'enseignement et de recherche français ou étrangers, des laboratoires publics ou privés. 


\title{
On Feedback Resource Allocation in Multiple-Input-Single-Output Systems Using Partial CSI Feedback
}

\author{
Behrooz Makki, Tommy Svensson, Senior Member, IEEE, Thomas Eriksson, and Mérouane Debbah, Fellow, IEEE
}

\begin{abstract}
This paper studies the problem of feedback resource allocation in multiple-input-single-output (MISO) channels utilizing partial channel state information (CSI) feedback. Considering low/moderate signal-to-noise ratios (SNRs), the optimal quantizers and the feedback bit allocation maximizing the throughput are obtained in the asymptotic case where the number of feedback bits increases. Moreover, the results are utilized to derive the optimal retransmission rates in the automatic repeat request (ARQ) protocols and joint CSI-ARQ schemes are proposed for the MISO setups. We show that uniform channel amplitude quantization is asymptotically optimal in terms of throughput. Also, the optimal retransmission rates of the incremental redundancy (INR) ARQ protocols follow an arithmetic progression in the exponential domain. Under certain conditions, a MISO system using quantized CSI can be mapped to a MISO or a SISO (S: single) setup using ARQ or joint CSI-ARQ feedback in the sense that they lead to the same throughput. Finally, to maximize the throughput, the optimal number of channel direction quantization bits should be $(M-1)$ times the number of amplitude quantization bits, where $M$ is the number of transmit antennas.
\end{abstract}

Index Terms-CSI quantization, HARQ feedback, MIMO transmission, throughput, joint phase-amplitude information feedback, incremental redundancy (INR) ARQ.

\section{INTRODUCTION}

D ESIGNING the optimal channel state information (CSI) quantization and feedback bit allocation for, e.g., maximizing the power-limited throughput is a complicated nonconvex problem which, depending on the fading model, may have no closed-form solution [1]-[3]. Particularly, the problem becomes more complex when the number of antennas at the transmitter(s) or receiver(s) increases. For this reason, most of the papers in the field of quantized CSI feedback focus on either channel amplitude quantization, e.g., [4], [5] or channel

Manuscript received May 30, 2014; revised September 25, 2014 and December 10, 2014; accepted January 22, 2015. This work was supported in part by the Swedish Governmental Agency for Innovation Systems (VINNOVA) within the VINN Excellence Center Chase. The editor coordinating the review of this paper and approving it for publication was M. C. Gursoy.

B. Makki, T. Svensson, and T. Eriksson are with the Department of Signals and Systems, Chalmers University of Technology, SE-412 96 Gothenburg, Sweden (e-mail: behrooz.makki@chalmers.se; tommy.svensson@ chalmers.se; thomase@chalmers.se).

M. Debbah is with Supelec, Plateau du Moulon, 91192 Gif-sur-Yvette, France (e-mail: merouane.debbah@supelec.fr).

Color versions of one or more of the figures in this paper are available online at http://ieeexplore.ieee.org.

Digital Object Identifier 10.1109/TCOMM.2015.2398861 direction quantization [6]-[15] and the quantization is normally based on random vector quantization or predefined tables of thresholds [13]-[16]. However, as also discussed in, e.g., [17][21], considerable performance improvement can be achieved when both partial channel direction and amplitude information are exploited for rate/power adaptation. On the other hand, feedback bit distribution is mostly based on simulations [22], [23] and the problem of joint CSI quantization and feedback bit allocation for maximizing the throughput has not been well formulated yet.

From another perspective, automatic repeat request (ARQ) is a sequential feedback approach to provide the transmitter with information about the channel quality. The optimization of the (re)transmission rates in the ARQ protocols is by itself a complex non-convex problem [2], [24]-[27]. However, as demonstrated in [2], [24] and in the following, under certain conditions the ARQ-based system can be mapped into an equivalent setup using quantized CSI feedback, in the sense that they lead to the same throughput. Thus, it is interesting to address the optimal CSI quantization and feedback bit distribution problem, because, as illustrated in the following, the results are useful not only for the quantized CSI schemes but also for deriving the optimal ARQ-based retransmission rates.

This paper studies the design of CSI quantization and feedback bit allocation in the multiple-input-single-output (MISO) setups. The optimization objective function is the system throughput. Also, the discussions are presented for the low/moderate signal-to-noise ratio (SNR) regimes and in the asymptotic conditions when the number of feedback bits increases. In the meantime, the analytical conclusions match with the numerical simulations with very high accuracy when tested for finite number of feedback bits. Moreover, we derive the optimal retransmission rates in the ARQ protocols, present joint CSI-ARQ approaches for the MISO networks and show conditions for when the quantized CSI, the ARQ and the joint CSI-ARQ feedback schemes can be mapped to each other.

The main conclusions of the paper are as follows:

- Considering low/moderate SNRs and product channel quantization codebooks with high number of feedback bits, uniform channel amplitude quantization is optimal in terms of throughput.

- The optimal low-SNR retransmission rates of the incremental redundancy (INR) ARQ protocols follow an arithmetic progression rule in the exponential domain when the number of retransmissions increases. 
- With proper setting of the number of quantization regions, the transmission power and the number of retransmissions, there exist mappings between the MISO and SISO (S: single) systems using joint channel direction and amplitude quantization, only channel amplitude quantization, the INR ARQ or joint CSI-INR ARQ feedback, in the sense that they result in the same throughput.

- With sufficiently large number of feedback bits and low/moderate SNRs, we have $\mathcal{Q}=(M-1) \mathcal{N}$ where $M$ is the number of transmit antennas and $\mathcal{Q}$ and $\mathcal{N}$ represent the optimal number of channel direction and amplitude feedback bits, respectively. Interestingly, our theoretical results are in harmony with the feedback bit allocation rules in, e.g., the 3GPP standards [1, Section IV.A].

Moreover, the numerical results indicate that the optimal channel amplitude quantization boundaries get closer to zero when the transmission power increases. Also, the efficiency of the uniform channel amplitude quantization is improved by increasing the number of transmit antennas. Finally, substantial throughput increment is achieved via very limited number of feedback bits, if the feedback signal is properly designed.

We should mention that the problem of CSI quantization has been previously studied in various papers, e.g., [2]-[21], but we develop new techniques and derive new conclusions that have not been presented before; as opposed to [2]-[16], we consider MISO setups, both the channel amplitude and direction quantizers are optimized in terms of throughput and we determine efficient feedback bit distribution between the channel direction and amplitude information maximizing the throughput. Moreover, the problem setup of the paper is different from the ones in [17]-[21] in which block diagnolization and predefined quantizers have been used for inter-cell interference suppression [17], numerical methods have been implemented to optimize (in terms of multiuser diversity gain) the number of quantization bits [18], a quantized CSI-based block diagonalization scheme is proposed for multiuser MIMO [19] and the feedback bit allocation has been optimized for outage-limited average power minimization [20], [21]. Finally, the discussions that we present about the ARQ and joint CSIARQ protocols have been considered by none of these papers.

\section{SySTEM MOdeL}

Consider a MISO system with $M$ transmit antennas which can be modeled as

$$
y=\sqrt{P} \mathbf{h}^{T} \mathbf{v} s+z .
$$

Here, $P$ is the transmission power, $\mathbf{h}=\left[h_{1} \ldots h_{M}\right]^{T} \in \mathcal{C}^{M}$ and $\mathbf{v}$ denote the fading random vector and the unit-norm beamforming vector, respectively, $s$ is the input signal and $z \in \operatorname{CN}(0,1)$ represents the white complex Gaussian noise added at the receiver. Also, $(.)^{T}$ denotes the transpose operator.

We study independent and identically distributed (iid) blockfading channels where the channel coefficients remain constant for a long time, determined by the channel coherence time, and then change according to their probability density functions (pdf:s). The results are obtained for the family of exponential distributions [28, Section 4.4] which can model most relevant fading conditions. Let $f_{G}$ and $F_{G}$ be the pdf and the cumulative distribution function (cdf) of a random variable in the family of exponential distributions. Among the properties of the family of exponential distributions are [28, Section 4.4]

$$
\begin{aligned}
& \forall g>0, \exists \mu_{1} \geq 0, \frac{\mathrm{d} f_{G}(g)}{\mathrm{d} g}<\mu_{1} \\
& \forall g>0, \exists \mu_{2} \geq 0, f_{G}(g)\left(\frac{\mathrm{d} f_{G}(g)}{\mathrm{d} g}\right)^{-1} \leq \mu_{2} .
\end{aligned}
$$

The receiver is assumed to have perfect CSI which is an acceptable assumption in block-fading channels [2]-[18], [20]. On the other hand, the transmitter is provided with quantized CSI (or ARQ feedback), as illustrated in the following. ${ }^{1}$ We concentrate on the continuous data communication model [2], [24]-[26] where a new codeword transmission starts as soon as the previous codeword transmission ends. Finally, the results are presented in natural logarithm basis and the throughput is given in nats-per-channel-use (npcu).

\section{Performance Analysis}

With the total of $B$ feedback bits, i.e., dividing $\mathrm{C}^{M}$ into $2^{B}$ quantization regions (QRs), the throughput of a MISO setup is obtained by

$$
\eta=\sum_{j=1}^{2^{B}} R_{j} \operatorname{Pr}\left(R_{j} \leq \log \left(1+P\left\|\mathbf{h}^{T} \mathbf{v}_{j}\right\|^{2}\right) \bigcap \mathbf{h} \in \xi_{j}\right)
$$

where $\mathbf{v}_{j}$ and $R_{j}$ denote the unit-norm beamforming vector and the data transmission rate considered for the $j$-th $\mathrm{QR}$, respectively, and $\xi_{j} \subset \mathrm{C}^{M}$ is the $j$-th $\mathrm{QR}$. Also, (4) is obtained by taking the expectation on the decodable data rates of all QRs.

To maximize the throughput, the boundaries of the QRs, the transmission rates $R_{j}$ and the beamforming vectors $\mathbf{v}_{j}$ should be optimized which, as stated before, cannot be determined with a closed-form solution. Therefore, suboptimal quantizers are normally considered and numerical solutions are utilized [4][18], [20], [21]. Here, we consider the class of product channel quantization codebooks which quantize the channel direction and amplitude information separately. Note that, while being suboptimal, the product quantization approach has some advantages such as lower storage requirements and faster quantization [20], [21], [29]. Also, many practical systems are equipped with channel amplitude quantizers [20]. Hence, the product quantization can be easily adopted in these systems.

With a product channel quantization approach, the channel amplitude and direction information are comprised as follows. Defining $G=\|\mathbf{h}\|^{2}$ as the squared channel amplitude, the distribution of the random variable $G$ is partitioned into $N$ QRs $\left[g_{n}, g_{n+1}\right)$, where $g_{n}$ 's are the amplitude quantization boundaries. Also, the channel direction information is quantized into $Q$ QRs with a uniform quantizer which is optimal for channel direction quantization in iid fading conditions. As examples, the 1- and the 2-dimensional representations of the QRs are

\footnotetext{
${ }^{1}$ The transmitter is assumed to know the long-term channel statistics, as it is required for parameter optimization.
} 


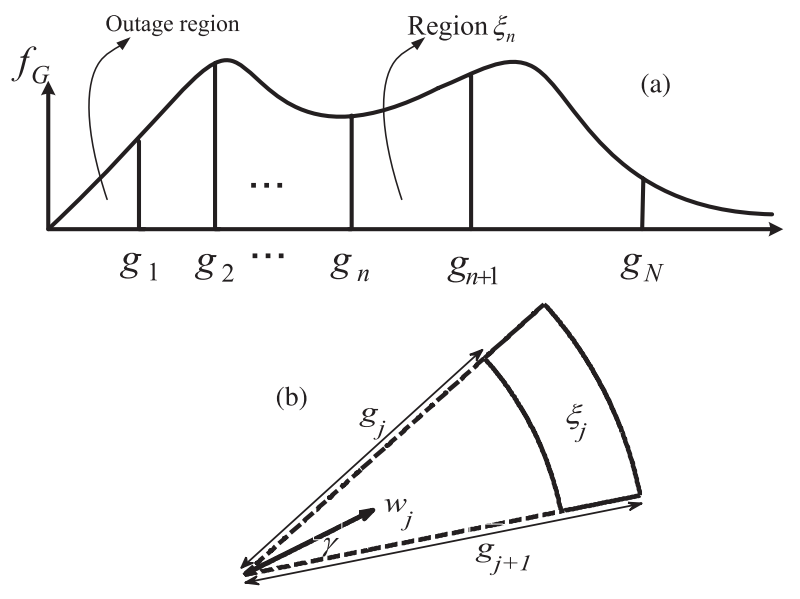

Fig. 1. The quantization boundaries in (a) a SISO and (b) a $2 \times 1$ MISO setup. With $M$ transmit antennas, the outage occurs iff the channel realization drops into the $M$-dimensional ball centered at the origin with radius $g_{1}$; otherwise the codeword is always decoded correctly.

represented in Fig. 1(a) and (b), respectively. Also, note that with the considered quantization scheme we have $N Q=2^{B}$.

Following the same procedure as in the SISO systems [2, Lemma 1], [3], it can be proved that the maximum throughput is achieved when the channel is assumed to be equal to its worst case in each QR. In this way, using (4), the maximum throughput is obtained by considering the beamformers according to

$$
\max _{\mathbf{v}_{j}} \inf _{\forall \mathbf{h} \in \xi_{j}}\left|\mathbf{h}^{T} \mathbf{v}_{j}\right|=\inf _{\forall \mathbf{h} \in \xi_{j}}\left|\mathbf{h}^{T} \mathbf{w}_{j}\right|=\sqrt{g_{j}} \cos \gamma
$$

where $\gamma$ is the angle of the QR (see Fig. 1(b) as an example). That is, the optimal beamformer in each QR is the unit-norm vector at the center of the region, denoted by $\mathbf{w}_{j}$, and the data transmission rate associated with the $j$-th $\mathrm{QR}$ is determined as

$$
R_{j}=\inf _{\forall \mathbf{h} \in \xi_{j}} \log \left(1+P\left|\mathbf{h}^{T} \mathbf{w}_{j}\right|^{2}\right)=\log \left(1+P g_{j} \cos ^{2} \gamma\right) .
$$

In this way, the outage occurs iff the channel realization drops into the $M$-dimensional ball centered at the origin with radius $g_{1}$; otherwise the codeword is always decoded correctly. Finally, due to the symmetry of the QRs in terms of direction parameters, the system throughput is found as

$$
\begin{aligned}
\eta & =\sum_{n=1}^{N} \log \left(1+P g_{n} \cos ^{2} \gamma\right) \operatorname{Pr}\left(G \in\left[g_{n}, g_{n+1}\right)\right) \\
& =\sum_{n=1}^{N} \log \left(1+P g_{n} \cos ^{2} \gamma\right)\left(F_{G}\left(g_{n+1}\right)-F_{G}\left(g_{n}\right)\right) .
\end{aligned}
$$

The objective of the paper is to maximize (7) subject to the total feedback budget $N Q=2^{B}$. Therefore, $N, Q$ and the quantization boundaries $g_{n}, \forall n$, should be properly designed. To achieve this goal, we consider the following procedure. First, we derive a closed-form relation between the quantization boundaries $g_{n}, \forall n$ (Lemma 1). The second step is to express the term $\cos ^{2} \gamma$, which represents the effect of quantized channel direction information, as a function of $Q$, i.e., the number of direction QRs. Here, we use the results of [11] leading to (13). Then, the results of Lemma 1 are utilized to write the quantization boundaries as a function of the number of amplitude QRs $N$. Lemmas 2 and 3 are dedicated to derive this function. Following the steps, (7) is rephrased as (17) which is a function of $N$ and $Q$. Therefore, adding the constraint $N Q=2^{B}$, we can determine the optimal feedback bit distribution rules, maximizing the throughput, as in (20). Indeed, to follow the steps, we need to use approximation and bounding techniques. In all bounding and approximation steps of the developed results, such as (12)-(14), and (19), we upper-bound the throughput. Also, we concentrate on the low-SNR regime with high resolution feedback, to keep the bounds/approximations reasonably tight. Moreover, as demonstrated in the following, the final conclusions are in harmony with the numerical simulations with high accuracy.

Let us first find a relationship between the quantization boundaries $g_{n}, \forall n$, as stated in Lemma 1 .

Lemma 1: With a product channel quantizer and low SNRs, the optimal, in terms of throughput, channel amplitude quantization tends towards uniform quantization, when the number of feedback bits increases asymptotically.

Proof: Setting $\frac{\partial \eta}{\partial g_{n}}=0$ in (7) leads to

$\frac{P \cos ^{2} \gamma\left(F_{G}\left(g_{n+1}\right)-F_{G}\left(g_{n}\right)\right)}{1+P \cos ^{2} \gamma g_{n}}+f_{G}\left(g_{n}\right) \log \left(\frac{1+P \cos ^{2} \gamma g_{n-1}}{1+P \cos ^{2} \gamma g_{n}}\right)=0$.

Assuming low $\mathrm{SNR}$ regimes, i.e., $P \rightarrow 0$, (8) is rephrased as

$$
F_{G}\left(g_{n+1}\right)-F_{G}\left(g_{n}\right)+f_{G}\left(g_{n}\right)\left(g_{n-1}-g_{n}\right)=0
$$

which leads to

$$
g_{n}=\frac{g_{n+1}+g_{n-1}}{2} .
$$

Here, we have used $\log (1+t) \rightarrow t$ for small values of $t$ and (10) follows from $\left(F_{G}\left(g_{n+1}\right)-F_{G}\left(g_{n}\right)\right) \rightarrow f_{G}\left(g_{n}\right)\left(g_{n+1}-g_{n}\right)$ for sufficiently large number of QRs. Thus, (10) indicates that, at low SNRs, the quantization boundaries follow an arithmetic progression. That is, the optimal, in terms of throughput, channel amplitude quantization tends towards uniform quantization, when the number of QRs increases.

Interestingly, the conclusion of the lemma is independent of the fading pdf and the transmission power as long as we are in low SNR regime and the number of QRs is high. Also, with uniform channel quantization we have

$$
g_{n}=g_{1}+(n-1) \alpha
$$

where $\alpha$ is the step size of the uniform quantization. Here, it is interesting to note that, although Lemma 1 proves the asymptotic optimality of the uniform quantizers in high-resolution feedback regimes, we still need to find the proper step size such that the entire range of positive values is covered by the quantizer and the gradient of the throughput, with respect to the quantization boundaries, goes to zero. The optimality condition of the step size is derived in Lemma 3 as explained in the following.

The following discussions are presented for the low SNR regime where, using (7), the normalized throughput function (normalized by the transmission power $P$ ) is given by

$$
\tilde{\eta}=\cos ^{2} \gamma \sum_{j=1}^{N} g_{j}\left(F_{G}\left(g_{j+1}\right)-F_{G}\left(g_{j}\right)\right) .
$$


This is an appropriate approximation at low SNRs (see [30] and the references therein). Also, note that the low-SNR regime is of interest in many communication scenarios such as wideband systems, sensor networks, cognitive radio, deep-space communications via satellites and communication at the edge of the cellular networks, e.g., [31], [32], where the available power per degree of freedom can be vanishingly small.

To follow the steps and rewrite (12) as a function of $Q$ and $N$, we use the bounds obtained in [11, eq. 15], i.e.,

$$
\begin{aligned}
(\sin \gamma)^{-2(M-1)}< & Q<\left(\frac{\sin \gamma}{2}\right)^{-2(M-1)} \\
& \Rightarrow 1-4 Q^{-\frac{1}{M-1}}<\cos ^{2} \gamma<1-Q^{-\frac{1}{M-1}}
\end{aligned}
$$

to upper bound the direction-related part of (12) as $\cos ^{2} \gamma<$ $1-Q^{-\frac{1}{M-1}}$. Then, Lemma 2 is utilized to upper bound the summation part of (12) which reflects the effect of channel amplitude quantization.

Lemma 2: The normalized throughput (12) is upper bounded by

$$
\begin{aligned}
& \frac{\tilde{\eta}}{\mathrm{E}\{G\} \cos ^{2} \gamma} \leq 1+\frac{\alpha(N-1)}{g_{1}} \\
&-\sum_{j=1}^{\infty}\left(\frac{-\alpha}{g_{1}}\right)^{j+1}\left\{\frac{1}{j+1} \sum_{k=0}^{j}\left(\begin{array}{c}
j+1 \\
k
\end{array}\right) B_{k}(N-1)^{j+1-k}\right\}
\end{aligned}
$$

where $B_{k}$ 's are the Bernoulli numbers, $\mathrm{E}\{$.$\} is the expectation$ operator and $\left(\begin{array}{l}n \\ k\end{array}\right)$ denotes the " $n$ choose $k$ " operator.

Proof: Using (12), the bound is obtained by

$$
\begin{aligned}
\frac{\tilde{\eta}}{\cos ^{2} \gamma} \stackrel{(a)}{=} g_{1} \operatorname{Pr}\left(G \geq g_{1}\right)+\alpha \sum_{n=2}^{N} \operatorname{Pr}\left(G \geq g_{n}\right) \\
\stackrel{(b)}{\leq} \mathrm{E}\{G\}\left(1+\sum_{n=2}^{N} \frac{\alpha}{g_{n}}\right) \\
\stackrel{(c)}{=} \mathrm{E}\{G\}\left(1+\frac{\alpha}{g_{1}} \sum_{n=1}^{N-1}\left(1+\sum_{j=1}^{\infty}(-1)^{j}\left(\frac{n \alpha}{g_{1}}\right)^{j}\right)\right) \\
=\mathrm{E}\{G\}\left(1+\frac{\alpha(N-1)}{g_{1}}-\sum_{j=1}^{\infty}\left(\frac{\alpha}{g_{1}}\right)^{j+1}\right. \\
\left.\times\left\{\frac{1}{j+1} \sum_{k=0}^{j}\left(\begin{array}{c}
j+1 \\
k
\end{array}\right) B_{k}(N-1)^{j+1-k}\right\}\right) .
\end{aligned}
$$

Here, $(a)$ comes from (11) and some manipulations and $(b)$ is based on Markov's inequality $\operatorname{Pr}(G \geq x) \leq \frac{\mathrm{E}\{G\}}{x}, x \geq 0[33$, p. 91]. Then, $(c)$ is obtained by (11) and Taylor expansion of the function $\frac{1}{1+x}$ and the last equality follows from some manipulations and the definition of Bernoulli numbers [34].

Lemma 2 rephrases the throughput as a function of the first quantization boundary $g_{1}$ and $\alpha$. Therefore, the next step to write (7) based on $Q$ and $N$ is to represent $\alpha$ as a function of $N$. The representation is derived in Lemma 3 as follows.
Lemma 3: With sufficiently high number of QRs and low SNRs, the optimal, in terms of throughput, quantization step size $\alpha$ is given by

$$
\alpha=N^{-\varepsilon(N)}, \lim _{N \rightarrow \infty} \varepsilon(N) \rightarrow 1
$$

where $\varepsilon(N)$ can be any function of the total number of QRs $N$.

Proof: The proof follows from the fact that, for every given power, a necessary condition for the optimality of the quantization approach is that the gradient of the throughput function with respect to the quantization boundaries goes to zero. Thus, (16) is obtained by finding the gradient of (12) with respect to $g_{n}$ 's, and letting it go to zero. More details can be found in the Appendix. ${ }^{2}$

Using (13), (14), and (16), the normalized throughput (12) is represented as

$$
\tilde{\eta} \leq \mathrm{E}\{G\}\left(1-Q^{-\frac{1}{M-1}}\right)
$$

$$
\begin{gathered}
\times\left(1+\frac{N^{-\varepsilon(N)}}{g_{1}}(N-1)-\sum_{j=1}^{\infty}\left(-\frac{N^{-\varepsilon(N)}}{g_{1}}\right)^{j+1}\right. \\
\left.\times\left\{\frac{1}{j+1} \sum_{k=0}^{j}\left(\begin{array}{c}
j+1 \\
k
\end{array}\right) B_{k}(N-1)^{j+1-k}\right\}\right)
\end{gathered}
$$

which is a function of $N$ and $Q$; this was our ultimate goal. Then, with a feedback budget constraint $N Q=2^{B}$, (17) is used to formulate the optimal, in terms of throughput, feedback bit distribution problem as

$$
\begin{aligned}
\max _{N, Q}\left\{( 1 - Q ^ { - \frac { 1 } { M - 1 } } ) \left(1+\frac{N^{-\varepsilon(N)}}{g_{1}}(N-1)-\sum_{j=1}^{\infty}\left(-\frac{N^{-\varepsilon(N)}}{g_{1}}\right)^{j+1}\right.\right. \\
\left.\left.\quad \times\left\{\frac{1}{j+1} \sum_{k=0}^{j}\left(\begin{array}{c}
j+1 \\
k
\end{array}\right) B_{k}(N-1)^{j+1-k}\right\}\right)\right\} \\
\text { s.t. } N Q=2^{B} .
\end{aligned}
$$

Using $B_{0}=1, B_{1}=\frac{1}{2}, \lim _{N \rightarrow \infty} \varepsilon(N) \rightarrow 1$ and the first-order approximation of the summation term in (17) by removing the terms $N^{-i}, i>1$, for large values of $N$, we take the logarithm of the objective function to rewrite (18) as

$$
\begin{aligned}
& \max _{N, Q}\left\{\log \left(1-\frac{1}{a N}\right)+\log \left(1-Q^{-\frac{1}{M-1}}\right)\right\} \\
& \qquad \stackrel{(d)}{=} \min _{N, Q}\left\{\frac{1}{a} N^{-1}+Q^{-\frac{1}{M-1}}\right\} \\
& \text { s.t. } N Q=2^{B}
\end{aligned}
$$

\footnotetext{
${ }^{2}$ Along with Lemma 3 , it is interesting to note that, with the family of exponential distributions and the optimal design of the high-resolution uniform quantizer, the expected overload distortion (related to the outer region of the quantizer) tends to zero faster than the granular distortion (corresponding to the in-region distortion) [35].
} 
where $a \doteq \frac{1+\frac{1}{s_{1}}-\sum_{j=1}^{\infty} \frac{1}{j+1}\left(\frac{-1}{g_{1}}\right)^{j+1}}{\frac{1}{g_{1}}-\frac{1}{2} \sum_{j=1}^{\infty}\left(\frac{-1}{g_{1}}\right)^{j+1}}$ and $(d)$ holds for large values of $N$ and $Q$ on which we focus. The optimal values of $N$ and $Q$ are obtained by setting $\frac{1}{a} N^{-1}=Q^{-\frac{1}{M-1}}, N Q=2^{B}$, which defining $Q \doteq \log _{2}(Q), \mathcal{N} \doteq \log _{2}(N)$ and $\mathcal{A} \doteq \log _{2}(a)$ leads to

$$
\begin{aligned}
\mathcal{Q} & =\frac{M-1}{M} B+\frac{M-1}{M} \mathcal{A}, \\
\mathcal{N} & =\frac{1}{M} B-\frac{M-1}{M} \mathcal{A} .
\end{aligned}
$$

The optimal numbers of bits are the closest integers to (20), (21). Interestingly, for large values of $B,(20),(21)$ result in

$$
\mathcal{Q}=(M-1) \mathcal{N} .
$$

That is, for sufficiently large number of feedback bits and low/moderate SNRs, the optimal number of quantization bits considered for channel direction information feedback tends to be $(M-1)$ times the number of feedback bits required for channel amplitude information feedback.

Regarding the derived bit allocation rule, the following points worth to be mentioned:

- The derived bit allocation rule is independent of the fading pdf and the transmission power as long as the transmission power is sufficiently low. Also, (22) holds for different step sizes as long as the optimality condition of Lemma 3 is satisfied.

- With a different amplitude quantization approach, the same bit allocation rule as in (22) has been previously derived by [20] for a different problem where the goal has been to design the power controllers for the MISO setups in outage-limited conditions. There, the optimal amplitude QRs minimizing the outage-limited transmission power have been shown to follow a geometric sequence, as opposed to our results with uniform channel amplitude quantization.

- Our theoretical conclusion is in line with, e.g., the standard 3GPP Release-99 where, with 2 transmit antennas, 2 and 3 feedback bits are considered for channel amplitude and direction quantization, respectively [1, Section IV.A] (With $M=2$ and $B=5,(20)$, (21) are rephrased as $Q=$ $2.5+0.5 \mathcal{A}, \mathcal{N}=2.5-0.5 \mathcal{A}$ which for the practical range of $\mathcal{A}$ lead to the integer numbers of bits $Q=3, \mathcal{N}=2$ ).

- We presented the analytical results for iid fading conditions, in harmony with [4], [5], [9]-[18], [20]. Limited feedback in correlated Rayleigh-fading MISO setups has been previously studied in, e.g., [6]-[8]. Particularly, as shown in [7], at low/moderate SNRs and with highresolution feedback, on which we focus, the capacity loss/decaying factor of the iid and correlated Rayleighfading channels, due to channel quantization, are the same and are given by [7, eq. (43)-(45)] which correspond to (13). Therefore, although we present the results for iid channels, (with high accuracy) the same bit allocation rule as in (22) holds for the correlated channels at low/moderate SNRs as well.

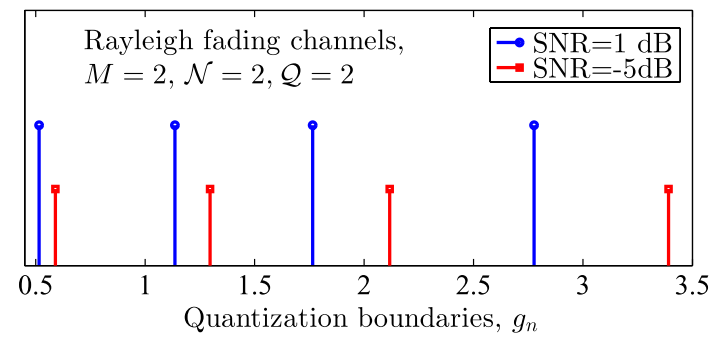

Fig. 2. The optimal quantization boundaries maximizing the throughput, Rayleigh-fading channel, $M=2, \mathcal{Q}=\mathcal{N}=2$. As demonstrated in the following, the results can be mapped to the optimal retransmission rates in a MISO setup utilizing joint CSI-INR ARQ with $Q$ channel direction feedback bits and a maximum of $I+1=2^{\mathcal{N}}$ INR-based retransmissions. Also, with proper scaling of the transmission SNR, the results of the figure give (1) the optimal retransmission rates in a MISO-INR ARQ setup utilizing perfect channel direction information and (2) the optimal QRs in a MISO setup with quantized channel amplitude and perfect channel direction information. See Theorem 1 and its following discussions for more details.

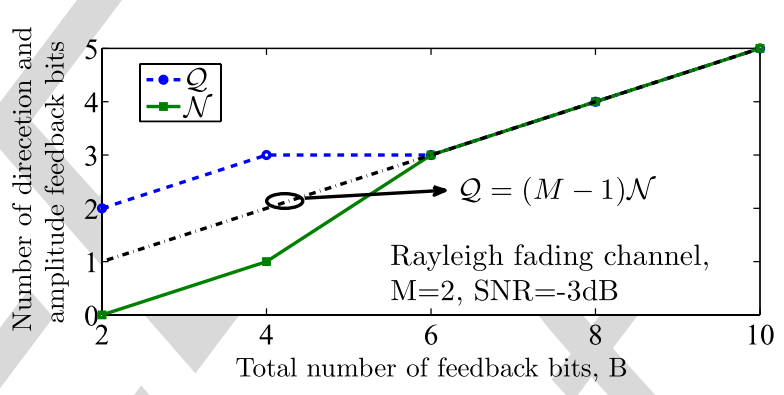

Fig. 3. The optimal, in terms of throughput, number of channel amplitude and direction feedback bits versus the total number of feedback bits $B$, Rayleighfading channel, $M=2, \mathrm{SNR}=-3 \mathrm{~dB}$. The results also give the optimal feedback resource allocation between the channel direction quantization and INR ARQ feedback in a joint CSI-INR ARQ feedback approach. See Theorem 1 and its following discussions for more details.

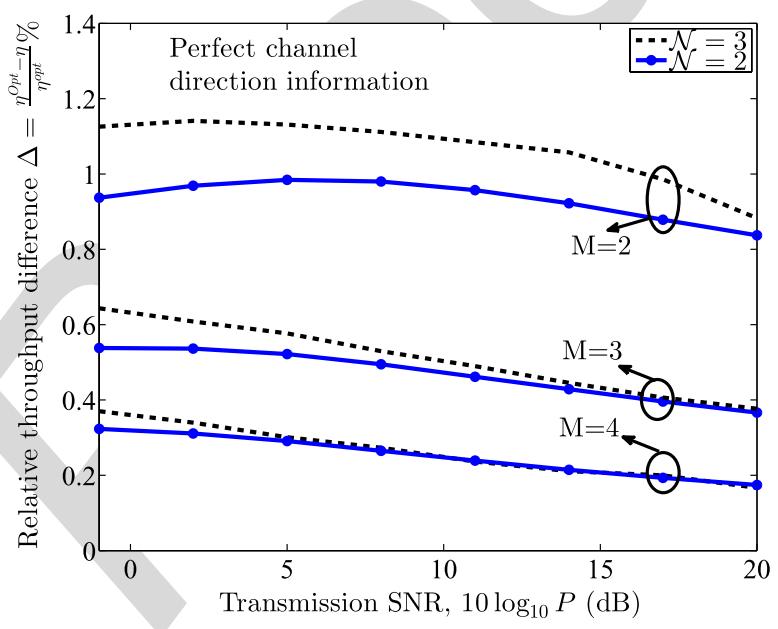

Fig. 4. The relative throughput difference $\Delta=\frac{\eta^{\text {opt }}-\eta}{\eta^{\text {opt }}} \%$ for different numbers of transmit antenna $M$, Rayleigh-fading channel. The results are obtained with perfect channel direction information at the transmitter, while they can be mapped to the cases with quantized channel direction feedback and scaled transmission SNRs (see Theorem 1).

- Our results are derived based on the high-resolution feedback assumption. However, as a well-known result in the source coding area, the high-resolution bounds agree well with the simulation results when tested for low resolutions, e.g., [7], [35] (see Figs. 2-6 as well). 


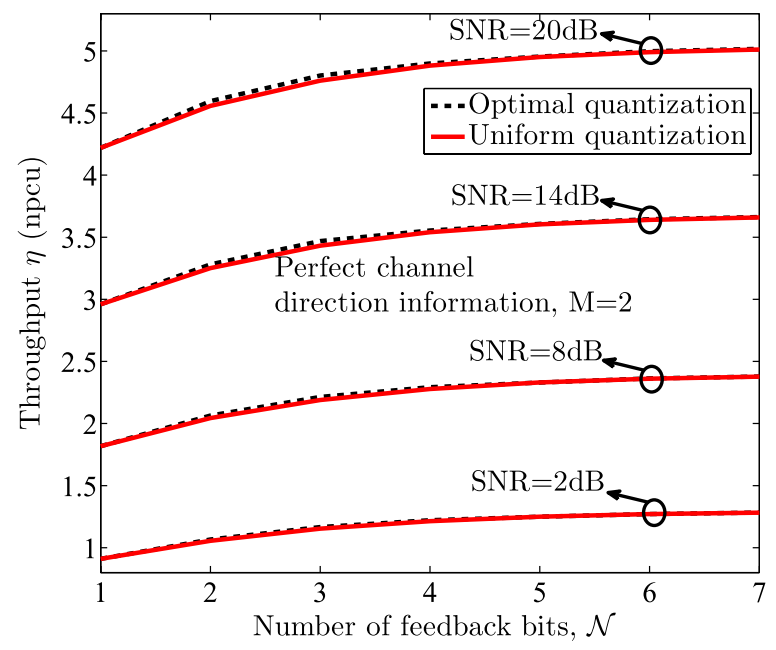

Fig. 5. The throughput for different transmit SNRs and numbers of amplitude feedback bits $\mathcal{N}$. Rayleigh-fading channel, $M=2$. The results are obtained with perfect channel direction information at the transmitter, while they can be mapped to the cases with quantized channel direction feedback and scaled transmission SNRs (see Theorem 1).

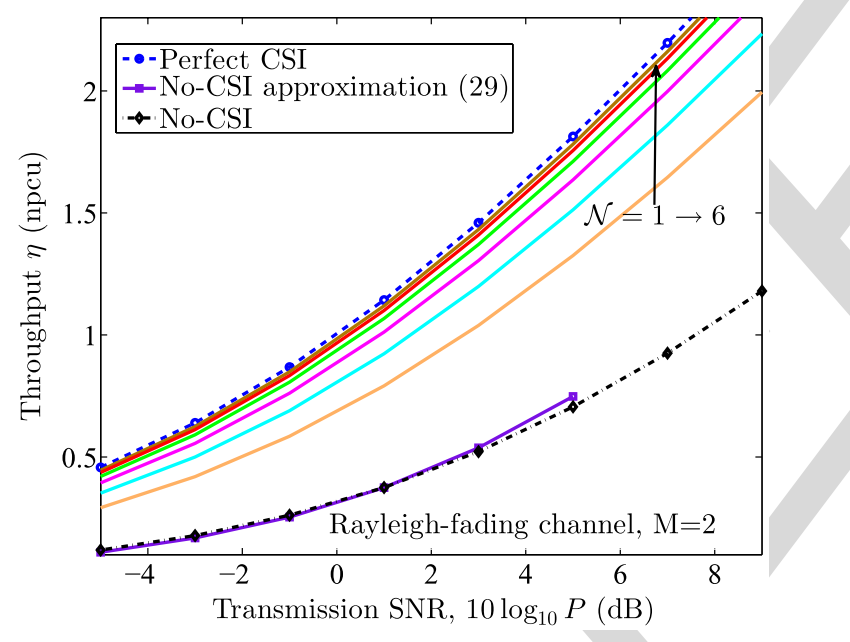

Fig. 6. The throughput in a Rayleigh-fading MISO channel with different levels of CSI feedback. The same throughput as in the cases with $\mathcal{N}$ channel amplitude feedback bits and perfect channel direction feedback is achieved when utilizing (1) both channel amplitude and direction quantization, (2) joint quantized channel direction information and INR ARQ feedback, and (3) only INR ARQ feedback, if the number of quantization bits, the transmission power and the number of ARQ retransmission rounds are adapted according to Theorem 1.

In Figs. 2-6, we evaluate the simulation results for Rayleighfading channels where each element of the channel vector $\mathbf{h}$ follows $\operatorname{eN}(0,1)$. Demonstrated in Fig. 2 are the optimal channel amplitude quantization boundaries for different transmission SNRs $10 \log _{10} P$ and $\mathcal{N}=\mathcal{Q}=2$. Also, setting $M=2$ and SNR $=-3 \mathrm{~dB}$, Fig. 3 shows the optimal bit distribution obtained by exhaustive search and compares the results with the ones obtained via our theoretical derivation (22). Here, the results are obtained for the optimal and uniform quantization approaches which, for the considered parameter setting of the figure, lead to the same optimal bit allocations demonstrated in the figure. The effect of different channel quantization schemes on the throughput is investigated in Figs. 4 and 5. Here, for different numbers of transmit antennas, the relative throughput difference function $\Delta=\frac{\eta^{\text {opt }}-\eta}{\eta^{\text {opt }}} \%$ is plotted where $\eta$ denotes the throughput achieved with uniform channel amplitude quantization and $\eta^{\text {opt }}$ is the maximum throughput achieved by exhaustive search on the amplitude QRs (Fig. 4). Also, considering perfect channel direction information, Fig. 5 shows the throughput for different quantization schemes and $M=2$. The results are obtained for different total numbers of feedback bits and transmission SNRs.

As demonstrated in the figures, the optimal channel amplitude quantization is very close to uniform quantization even for limited number of feedback bits (Fig. 2). However, the last quantization boundary of the optimal quantizer grows large, as expected. Moreover, the optimal channel amplitude quantization boundaries get closer to zero when the transmission power increases (Fig. 2). The theoretical results of (20)-(22) are in harmony with the optimal feedback bit allocation rules derived via simulations, when the total number of feedback bits increases (Fig. 3). Also, it is interesting to note that optimizing the throughput at high SNRs we observe the optimal number of channel direction quantization bits to be higher (or equal) than the ones allocated for amplitude quantization. However, depending on the number of antennas and the SNR, different optimal bit allocations are obtained at high SNRs; this is expected because our results have not been proved for high SNRs. In Fig. 4, we observe higher relative throughput difference in the cases with $\mathcal{N}=3$, compared to the case of $\mathcal{N}=2$, which is because of the finite number of feedback bits and the range of SNR in the figure. On the other hand, the efficiency of the uniform channel amplitude quantization is improved by increasing the number of transmit antennas/feedback bits, and the difference between the throughput of the optimal and uniform quantization-based schemes is negligible even with limited number of feedback bits (Figs. 4 and 5). Particularly, for a large range of SNRs the relative throughput difference is less than $1 \%$ (Fig. 4).

To close the discussions, we should mention that throughout the paper we concentrate on the single-user MISO setups. Our reasons for selecting the MISO systems are 1) different standards such as the $3 \mathrm{GPP}[1$, Section IV.A] and the $3 \mathrm{GPP}$ LTE [1, Section IV.D], [36] mainly concentrate on the limited feedback schemes for the MISO setups, 2) the analytical results can be derived/followed, 3) a large portion of the related literature is devoted to the MISO systems, e.g., [6]-[8], [10], [14], [20], [36]. However, our results also provide some insights/tools for the cases with multiuser MIMO. An interesting extension of the paper, in which we are currently involved, is to study the feedback distribution in multiuser systems. Intuitively, we expect the same conclusions as in the single-user MISO setup, when we extend the results to the multiuser systems. Particularly, considering the outage-limited power minimization problem in the multiuser setups, [21] shows the same feedback bit distribution rule as in (22). Finally, deriving feedback bit distribution rules based on the lower bounds of the throughput is an interesting topic for extensions. 


\section{A. Relevance to the ARQ-Based Schemes}

It has been previously demonstrated that the throughput of a system utilizing INR ARQ protocol with a maximum of $I+1$ (re)transmission rounds, continuous data communication and uniform power allocation is obtained by [2], [24] ${ }^{3}$

$$
\eta^{\mathrm{INR}}=\sum_{i=1}^{I+1} R_{i}^{\mathrm{INR}}\left(F_{G}\left(\frac{e^{R_{i-1}^{\mathrm{INR}}}-1}{P}\right)-F_{G}\left(\frac{e^{R_{i}^{\mathrm{INR}}}-1}{P}\right)\right) .
$$

Here, $R_{i}^{\mathrm{INR}}, R_{0}^{\mathrm{INR}} \doteq \infty$, is the equivalent data rate at the end of the $i$-th round. Then, with a SISO setup, [2, Theorem 1], [24, Lemma 3] have used (23) to relate the throughput of the ARQ-based scheme to the throughput in the quantized CSI-based approach. The following theorem extends the results of [2], [24] to a MISO setup. Moreover, we derive the optimal, in terms of throughput, rate allocation of the INR ARQ and propose joint CSI-ARQ protocols which lead to the same throughput as in the cases with only CSI quantization. The theorem is of interest because 1) it uses the results of the quantized CSI scheme to solve the problem of optimal retransmission rate allocation in the MISO-INR systems; the problem which is complex non-convex in general. Moreover, 2) there are many papers using only channel amplitude or channel direction quantization. Then, the theorem provides connections between the papers considering one of these schemes.

Theorem 1: The following assertions are valid:

a) Maximizing the low-SNR throughput with asymptotically high number of retransmissions, the equivalent data rates of the INR protocol follow an arithmetic progression in the exponential domain.

b) In the optimal case, the same throughput is achieved in the following scenarios:

- Scenario 1: A MISO setup with product CSI quantization consisting of $N$ and $Q$ QRs for the channel amplitude and direction quantization, respectively, and transmission power $P$.

- Scenario 2: A MISO setup utilizing joint CSI-ARQ feedback as follows. First, a uniform channel direction quantization with $Q$ QRs is used to inform the transmitter about the channel direction. Then, with no pre-knowledge about the channel amplitude, the data is transmitted via an INR ARQ with a maximum of $I+1=N$ retransmissions and power $P$.

- Scenario 3: A MISO-INR approach with a maximum of $I+1=N$ retransmissions, perfect channel direction information and transmission power $P \cos ^{2} \gamma$ with $\gamma$ obtained in Scenario 1.

- Scenario 4: A MISO setup with $N$ channel amplitude QRs, perfect channel direction information and transmission power $P \cos ^{2} \gamma$ with $\gamma$ obtained in Scenario 1.

In all considered scenarios, the throughput is given by (7).

${ }^{3}$ In [2], [24], the SISO model is considered. But, the same argument as in [2], [24] is valid for the MISO setup too.
Proof: Using the variable transformation $R_{I+2-i}^{\mathrm{INR}} \dot{=}$ $\log \left(1+P g_{i}\right)$ and replacing $P \cos ^{2} \gamma$ by $P,(23)$ is mapped to (7) with $N=I+1$. Therefore, following the same arguments as in Lemma 1, we have

$$
e^{R_{i}^{\mathrm{INR}}}=\frac{e^{R_{i+1}^{\mathrm{INR}}}+e^{R_{i-1}^{\mathrm{INR}}}}{2}
$$

in the optimal case, which follows from (10) and $R_{I+2-i}^{\mathrm{INR}} \dot{=}$ $\log \left(1+P g_{i}\right)$. That is, at low SNRs, the equivalent data rates of the INR protocol follow an arithmetic progression in the exponential domain, as stated in part (a) of the theorem.

For part (b), the proof follows from (7) and (23) where, using the variable transform $R_{I+2-i}^{\mathrm{INR}} \doteq \log \left(1+P g_{i}\right)$ and replacing $P \cos ^{2} \gamma$ by $P$ whenever required, it can be shown that the same throughput is achieved in all considered scenarios. Note that, in contrast to part (a), there is no approximation in part (b) and the conclusion holds for every transmission power/number of QRs.

We should mention that the result of Theorem 1 part (a) is different from the one in [25, Theorem 1] which studies the performance of INR ARQ protocols in the bursty data communication models. There, the optimal retransmission rates have been shown to follow a geometric sequence $R_{i}^{\mathrm{INR}}=$ $\sqrt{R_{i-1}^{\mathrm{INR}} R_{i+1}^{\mathrm{INR}}}$, as opposed to the conclusion of Theorem 1 part (a) with $e^{R_{i}^{\mathrm{INR}}}=\frac{e_{i+1}^{R_{i+1}^{\mathrm{NR}}}+e_{i-1}^{R_{i-1}}}{2}$. As discussed in [24], [25], there are fundamental differences between the performance of ARQ protocols in the bursty and continuous data communication models. Specially, the ARQ-based throughput achieved in these data communication models are different which results in differences in the optimal retransmission rates.

Following the arguments of Theorem 1, (22) gives the optimal feedback resource allocation between the channel direction quantization feedback bits and the INR-based ARQ feedback bits in the joint CSI-ARQ model of Theorem 1 (see Fig. 3). Moreover, using $R_{I+2-i}^{\mathrm{INR}} \doteq \log \left(1+P g_{i}\right)$, Fig. 2 gives the optimal INR-based retransmission rates of the considered joint CSIARQ approach with $Q=2$. Also, with proper scaling of the transmission SNR, the results of the figure give 1) the optimal retransmission rates in a MISO-INR ARQ setup utilizing perfect channel direction information and 2) the optimal quantization boundaries in a MISO setup with quantized channel amplitude and perfect channel direction information.

Theorem 1 presented equivalent models for different MISO setups, while there are also mappings between the MISO and SISO systems utilizing partial CSI feedback. For instance, the throughput achieved in a MISO setup with $N$ and $Q$ channel amplitude and direction QRs, respectively, is the same as the throughput achieved in a SISO system with $N$ channel amplitude QRs, transmission power $P \cos ^{2} \gamma$ and channel gain cdf $F_{G}$. This is because in both cases the throughput is given by (7). Finally, Theorem 1 proposed different joint CSI-ARQ, ARQ, quantized CSI with channel direction and amplitude (or each one alone) feedback models which lead to the same throughput. Then, selecting the best scheme depends on many parameters such as the implementation complexity, delay requirement and other protocol aspects. 
Fig. 6 demonstrates the system throughput in a $2 \times 1$ Rayleigh-fading MISO setup. Here, considering $\mathcal{N}=1, \ldots, 6$ number of amplitude feedback bits, the throughput is obtained with uniform channel amplitude quantization, and the results are compared with cases having perfect/no CSI feedback. Again, we can follow the discussions of Theorem 1 to map the results to the cases with different quantized CSI, ARQ or joint CSI-ARQ feedback models. Also, the difference between the throughput of the optimal and uniform quantizationbased schemes is negligible for the considered range of SNR. Thus, we only plot the results of the uniform quantizationbased scheme. Note that for Rayleigh fading conditions we have $f_{G}(x)=\frac{1}{\Gamma(M)} x^{M-1} e^{-x}$ and $F_{G}(x)=1-\frac{\Gamma(M, x)}{\Gamma(M)}, x \geq 0$, with $\Gamma(x)$ and $\Gamma(m, x)$ representing the Gamma and the incomplete Gamma functions, respectively. Therefore, the perfectCSI throughput presented in Fig. 6 is obtained by

$$
\begin{aligned}
\eta^{\text {perfect }} & =\int_{0}^{\infty} \frac{1}{\Gamma(M)} x^{M-1} e^{-x} \log (1+P x) \mathrm{d} x \\
& \stackrel{(e)}{=} \frac{1}{\Gamma(M)} \sum_{j=1}^{\infty} \frac{(-1)^{j+1} P^{j}}{j} \int_{0}^{\infty} e^{-x} x^{M+j-1} \mathrm{~d} x \\
& =\frac{1}{\Gamma(M)} \sum_{j=1}^{\infty} \frac{(-1)^{j} P^{j}}{j} \Gamma(M+j)
\end{aligned}
$$

where $(e)$ comes from the Taylor expansion of $\log (1+P x)$. Also, the no-CSI throughput is given by

$$
\begin{aligned}
\eta^{\mathrm{No}} & =\max _{R}\left\{R\left(1-F_{G}\left(\frac{M\left(e^{R}-1\right)}{P}\right)\right)\right\} \\
& =\max _{x}\left\{\log \left(1+\frac{P}{M} x\right)\left(1-F_{G}(x)\right)\right\} \\
& =\frac{1}{\Gamma(M)} \max _{x}\left\{\log \left(1+\frac{P}{M} x\right) \Gamma(M, x)\right\}
\end{aligned}
$$

which, depending on the value of $M$, may have no closedform solution. ${ }^{4}$ However, as the first-order approximation, we can use $\log \left(1+\frac{P}{M} x\right) \approx \frac{P}{M} x$ and $\Gamma(M, x) \approx(M-1) !-\frac{x^{M}}{M}$ to rephrase the maximization problem of (26) as

$$
\begin{aligned}
\frac{1}{\Gamma(M)} \max _{x}\left\{\log \left(1+\frac{P}{M} x\right) \Gamma(M, x)\right\} & \\
& \approx \frac{P}{M^{2} \Gamma(M)} \max _{x}\left\{x\left(M !-x^{M}\right)\right\}
\end{aligned}
$$

and find a low-SNR approximation of the no-CSI throughput as

$$
\eta^{\text {No }} \approx \log \left(1+\frac{P}{M} \sqrt[M]{\frac{M !}{M+1}}\right) \frac{\Gamma\left(M, \sqrt[M]{\frac{M !}{M+1}}\right)}{\Gamma(M)} .
$$

\footnotetext{
${ }^{4}$ Setting $M=1$ as a special case, the no-CSI throughput of a SISO setup is given by

$$
\eta^{\mathrm{No}}=\max _{x}\left\{e^{-x} \log (1+x P)\right\}=\Lambda(P) e^{-\frac{e^{\Lambda(P)}-1}{P}}
$$

where $\Lambda($.) denotes the Lambert $\mathrm{W}$ function.
As shown in Fig. 6, the approximation (29) is tight at low SNRs and the tightness decreases with the SNR. Also, considering different transmission SNRs, substantial throughput increment is observed with limited number of amplitude QRs.

\section{CONCLUSION}

This paper studied the design of optimal CSI quantization and feedback bit allocation in MISO systems when the number of feedback bits increases. Also, we presented discussions on how to select the (re)transmission rates of the INR ARQ protocol such that the throughput is maximized. In terms of throughput, the results showed that: 1) uniform quantization is asymptotically optimal for MISO channels. 2) The INR-based retransmission rates follow an arithmetic progression in the exponential domain. 3) For sufficiently large number of feedback bits, the optimal number of channel direction quantization bits tends to be $(M-1)$ times the number of channel amplitude quantization bits where $M$ is the number of transmit antennas. Finally, 4) there exist mappings between the throughput of the MISO systems utilizing INR ARQ, joint CSI-ARQ or different quantized CSI schemes.

\section{APPENDIX}

\section{A. Proof of Lemma 3}

Using (12) with uniform amplitude quantization, we derive the gradient of the throughput as

$$
\begin{gathered}
\left\{\begin{aligned}
\left|\frac{\partial \tilde{\eta}}{\partial g_{n}}\right| & =\left|\int_{g_{n}}^{g_{n+1}} f_{G}(t) \mathrm{d} t-f_{G}\left(g_{n}\right)\left(g_{n-1}-g_{n}\right)\right| \\
& \stackrel{(f)}{\leq} \frac{1}{2} \max _{x \in\left[g_{n}, g_{n+1}\right]}\left(\frac{\mathrm{d} f_{G}(x)}{\mathrm{d} x}\right) \alpha^{2} \\
\left|\frac{\partial \tilde{\eta}}{\partial g_{N}}\right| & =\left|f_{G}\left(g_{N}\right)\left(g_{n-1}-g_{N}\right)+1-f_{G}\left(g_{N}\right)\right| \\
& =\left|1-f_{G}\left(g_{N}\right)-\alpha f_{G}\left(g_{N}\right)\right| \doteq \Phi
\end{aligned}\right. \\
\begin{aligned}
\|\nabla\{\tilde{\eta}\}\| & =\left(\sum_{\forall g_{n}}\left|\frac{\partial \tilde{\eta}}{\partial g_{n}}\right|^{2}\right)^{\frac{1}{2}} \leq \Phi+\left(\sum_{\forall g_{n}, n \neq N}\left|\frac{\partial \tilde{\eta}}{\partial g_{n}}\right|^{2}\right)^{\frac{1}{2}}
\end{aligned} \\
\leq \Phi+\frac{1}{2} \mu_{1} \alpha^{2} \sqrt{N} .
\end{gathered}
$$

Here, $(f)$ is obtained by the second-order expansion of the function $y(x)=\int_{a}^{x} f(t) \mathrm{d} t$ and the fact that with uniform channel quantization we have $g_{n}=g_{1}+(n-1) \alpha$. Moreover, (30.II) comes from $g_{n}=g_{1}+(n-1) \alpha, \forall n$. The first inequality in (30.III) is based on triangle inequality. Also, (g) follows from (30.I) and defining $\mu_{1} \doteq \max _{x \geq 0}\left\{\frac{\mathrm{d} f_{G}(x)}{\mathrm{d} x}\right\}$ (see (2) and (3) for the properties of the family of exponential distributions).

For the asymptotic optimality of the uniform amplitude quantizer, the necessary condition is that the gradient (30.III) goes to zero when $N$ increases. We use this condition and (30.III) to derive (16) as follows. For the family of exponentially-distributed 
variables, the term $\Phi=f_{G}\left(g_{N}\right)\left|\alpha-\frac{\int_{g_{N}}^{\infty} f_{G}(t) \mathrm{d} t}{f_{G}\left(g_{N}\right)}\right|$ in (30.II) goes to zero exponentially if $g_{N} \rightarrow \infty$. This is because using L'Hopital's rule we have

$\Phi=f_{G}\left(g_{N}\right)\left|\alpha+f_{G}(g)\left(\frac{d f_{G}(g)}{d g}\right)^{-1}\right| \leq f_{G}\left(g_{N}\right)\left(|\alpha|+\left|\mu_{2}\right|\right)$

if $g_{N} \rightarrow \infty$ (see (3)). On the other hand, for $g_{N}=g_{1}+(N-$ 1) $\alpha \rightarrow \infty$ and vanishing gradient, we should have $\alpha \geq \mathcal{O}\left(N^{-1}\right)$ for the step size where $\mathcal{O}(X)$ denotes the order of the function $X$. Therefore, in the extreme case we have

$$
\alpha=N^{-\varepsilon(N)}, \lim _{N \rightarrow \infty} \varepsilon(N) \rightarrow 1, \varepsilon(N) \leq 1, \forall N
$$

which leads to $\frac{1}{2} \mu_{1} \alpha^{2} \sqrt{N}=\mathcal{O}\left(N^{-\frac{3}{2}}\right)$ and, from (30.III),

$$
\|\nabla\{\tilde{\eta}\}\| \leq \mathcal{O}\left(e^{-N}\right)+\mathcal{O}\left(N^{-\frac{3}{2}}\right)=\mathcal{O}\left(N^{-\frac{3}{2}}\right) \rightarrow 0
$$

when $N$ increases. Finally, using the step size (32), we have $g_{n}=g_{1}+(n-1) N^{-\varepsilon(N)}$ and $\frac{g_{n}}{g_{n+1}} \rightarrow 1$ for large $n$ 's. Moreover, while (32) gives the optimality condition of the step size, the codebook that achieves the optimal scaling is not unique, and different step sizes can be determined as long as (32) is satisfied (for more discussions and some examples of proper quantization step sizes with asymptotically high number of quantization regions, see [20, Section II.C] as well).

\section{REFERENCES}

[1] D. J. Love et al., "An overview of limited feedback in wireless communication systems," IEEE J. Sel. Areas Commun., vol. 26, no. 8, pp. 13411365, Oct. 2008

[2] B. Makki and T. Eriksson, "On hybrid ARQ and quantized CSI feedback schemes in quasi-static fading channels," IEEE Trans. Commun., vol. 60, no. 4, pp. 986-997, Apr. 2012.

[3] T. T. Kim and M. Skoglund, "On the expected rate of slowly fading channels with quantized side information," IEEE Trans. Commun., vol. 55, no. 4, pp. 820-829, Apr. 2007.

[4] T. Kim and M. Skoglund, "Diversity-multiplexing tradeoff in MIMO channels with partial CSIT," IEEE Trans. Inf. Theory, vol. 53, no. 8, pp. 2743-2759, Aug. 2007.

[5] B. Makki, J. Li, T. Eriksson, and T. Svensson, "Throughput analysis for multi-point joint transmission with quantized CSI feedback," in Proc. IEEE VTC Fall, Sep. 2012, pp. 1-5.

[6] Y. Isukapalli and B. D. Rao, "Finite rate feedback for spatially and temporally correlated MISO channels in the presence of estimation errors and feedback delay," in Proc. IEEE GLOBECOM, Nov. 2007, pp. 2791-2795.

[7] J. Zheng and B. D. Rao, "Analysis of multiple antenna systems with finite-rate channel information feedback over spatially correlated fading channels," IEEE Trans. Signal Process., vol. 55, no. 9, pp. 4612-4626, Sep. 2007.

[8] J. Zheng and B. D. Rao, "Capacity analysis of correlated multiple antenna systems with finite rate feedback," in Proc. IEEE ICC, Jun. 2006, vol. 9, pp. 4173-4179.

[9] W. Xu, C. Zhao, and Z. Ding, "Limited feedback design for MIMO broadcast channels with ARQ mechanism," IEEE Trans. Wireless Commun., vol. 8, no. 4, pp. 2132-2141, Apr. 2009.

[10] W. Xu, C. Zhao, and Z. Ding, "Optimisation of limited feedback design for heterogeneous users in multi-antenna downlinks," IET Commun., vol. 3 , no. 11, pp. 1724-1735, Nov. 2009.
[11] D. J. Love, R. W. Heath, and T. Strohmer, "Grassmannian beamforming for multiple-input multiple-output wireless systems," IEEE Trans. Inf. Theory, vol. 49, no. 10, pp. 2735-2747, Oct. 2003.

[12] S. Zhou, J. Gong, and Z. Niu, "Distributed adaptation of quantized feedback for downlink network MIMO systems," IEEE Trans. Wireless Commun., vol. 10, no. 1, pp. 61-67, Jan. 2011.

[13] N. Jindal, "MIMO broadcast channels with finite-rate feedback," IEEE Trans. Inf. Theory, vol. 52, no. 11, pp. 5045-5060, Nov. 2006.

[14] R. Bhagavatula and R. W. Heath, "Adaptive limited feedback for sum-rate maximizing beamforming in cooperative multicell systems," IEEE Trans. Signal Process., vol. 59, no. 2, pp. 800-811, Feb. 2011.

[15] J. Zhang, M. Kountouris, J. G. Andrews, and R. W. Heath, "Achievable throughput of multi-mode multiuser MIMO with imperfect CSI constraints," in Proc. IEEE ISIT, Jul. 2009, pp. 2659-2663.

[16] L. Li, M. Pesavento, and A. B. Gershman, "Downlink opportunistic scheduling with low-rate channel state feedback: Error rate analysis and optimization of the feedback parameters," IEEE Trans. Commun., vol. 58, no. 10, pp. 2871-2880, Oct. 2010.

[17] J. Jin, Q. Wang, C. Lin, H. Yang, and Y. Wang, "Coordinated multipoint transmission with limited feedback," in Proc. IEEE GLOBECOM, Dec. 2010, pp. 850-854.

[18] M. Kountouris, R. de Francisco, D. Gesbert, D. T. M. Slock, and T. Salzer, "Multiuser diversity-multiplexing tradeoff in MIMO broadcast channels with limited feedback," in Proc. Asilomar, Nov. 2006, pp. 364-368

[19] S. H. Moon, S. R. Lee, J. S. Kim, and I. Lee, "Channel quantization for block diagonalization with limited feedback in multiuser MIMO downlink channels," J. Commun. Netw., vol. 16, no. 1, pp. 1-9, Feb. 2014.

[20] B. Khoshnevis and W. Yu, "Bit allocation law for multiantenna channel feedback quantization: Single-user case," IEEE Trans. Signal Process., vol. 59, no. 5, pp. 2270-2283, May 2011.

[21] B. Khoshnevis and W. Yu, "Bit allocation laws for multiantenna channel feedback quantization: Multiuser case," IEEE Trans. Signal Process., vol. 60, no. 1, pp. 367-382, Jan. 2012.

[22] "System level comparison of best-M and DCT-based CQI compression schemes," Huawei, RAN1 Meeting 47bis, Sorrento, Italy, R1-070368, Jan. 15-19, 2007. [Online]. Available: http://www.3gpp.org/DynaReport/ TDocExMtg--R1-47b--26241.htm

[23] B. Makki and T. Eriksson, "Efficient channel quality feedback signaling using transform coding and bit allocation," in Proc. IEEE 71st VTC Spring, May 2010, pp. 1-5.

[24] C. Shen, T. Liu, and M. P. Fitz, "On the average rate performance of hybrid-ARQ in quasi-static fading channels," IEEE Trans. Commun., vol. 57, no. 11, pp. 3339-3352, Nov. 2009.

[25] B. Makki and T. Eriksson, "On the performance of MIMO-ARQ systems with channel state information at the receiver," IEEE Trans. Commun., vol. 62, no. 5, pp. 1588-1603, May 2014.

[26] C. Shen and M. P. Fitz, "Hybrid ARQ in multiple-antenna slow fading channels: Performance limits and optimal linear dispersion code design," IEEE Trans. Inf. Theory, vol. 57, no. 9, pp. 5863-5883, Sep. 2011.

[27] L. Szczecinski, C. Correa, and L. Ahumada, "Variable-rate transmission for incremental redundancy hybrid ARQ," in Proc. IEEE GLOBECOM, Dec. 2010, pp. 1-5.

[28] J. L. Devore, Probability and Statistics for Engineering and the Sciences, 8th ed. Pacific Grove, CA, USA: Duxbury, 2011.

[29] K. Sayood, Introduction to Data Compression, 3rd ed. San Mateo, CA, USA: Morgan Kaufmann, 2006.

[30] V. Prelov and S. Verdu, "Second-order asymptotics of mutual information," IEEE Trans. Inf. Theory, vol. 50, no. 8, pp. 1567-1580, Aug. 2004.

[31] L. Sboui, Z. Rezki, and M. Alouini, "Achievable rate of spectrum sharing cognitive radio systems over fading channels at low power regime," IEEE Trans. Wireless Commun., vol. 13, no. 11, pp. 6461-6473, Nov. 2014.

[32] X. Li, S. Jin, M. R. McKay, X. Gao, and K. K. Wong, "Capacity of MIMOMAC with transmit channel knowledge in the low SNR regime," IEEE Trans. Wireless Commun., vol. 9, no. 3, pp. 926-931, Mar. 2010.

[33] E. M. Stein and R. Shakarchi, Real Analysis: Measure Theory, Integration, Hilbert Spaces. Princeton, NJ, USA: Princeton Univ. Press, 2008.

[34] T. Agoh and K. Dilcher, "Reciprocity relations for Bernoulli numbers," Amer. Math. Monthly, vol. 115, no. 3, pp. 237-244, 2008

[35] T. Eriksson and E. Agrell, "Lattice-Based Quantization part II," Dept. Inf. Theory, Chalmers Univ. Technol., Goteborg, Sweden, Oct. 18, 1996, Tech. Rep.

[36] J. Mirza, P. A. Dmochowski, P. J. Smith, and M. Shafi, "Limited feedback multiuser MISO systems with differential codebooks in correlated channels," in Proc. IEEE ICC, Jun. 2013, pp. 5386-5391. 


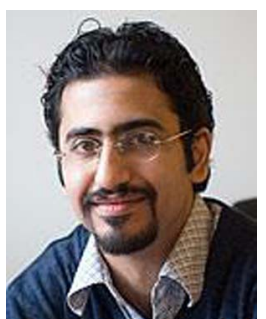

Behrooz Makki was born in Tehran, Iran. He received the B.Sc. degree in electrical engineering from Sharif University of Technology, Tehran, Iran, and the M.Sc. degree in bioelectric engineering from Amirkabir University of Technology, Tehran, Iran, respectively. He received the $\mathrm{Ph} . \mathrm{D}$. degree in communication engineering from Chalmers University of Technology, Gothenburg, Sweden. Since 2013, he has been working as a Postdoc at Chalmers University. His current research interests include partial channel state information (CSI) feedback, hybrid automatic repeat request, green communication, correlated fading channels, spectrum sharing, relay, satellite, and CoMP networks.

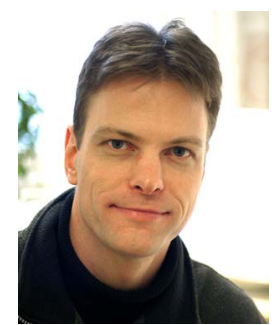

Tommy Svensson (S'98-M'03-SM'10) received the Ph.D. degree in information theory from Chalmers University of Technology in 2003, and he has worked at Ericsson $\mathrm{AB}$ with core networks, radio access networks, and microwave transmission products. He is Associate Professor in Communication Systems at Chalmers University, where he is leading the research on air interface and wireless backhaul networking technologies for future wireless systems. He was involved in the European collaborative research projects WINNER I, II, WINNER+, and ARTIST4G, which have contributed to the 3GPP LTE standards, and he is currently active in the EU FP7 METIS project targeting solutions for $5 \mathrm{G}$. His main research interests are in design and analysis of physical layer algorithms, multiple access, resource allocation, cooperative systems, moving relays/cells/networks, as well as satellite systems. He has co-authored two books and more than 100 journal and conference papers. He is chairman of the IEEE Sweden Vehicular Technology/Communications/Information Theory Chapter, and Coordinator of the Communication Engineering Master's Program at Chalmers.

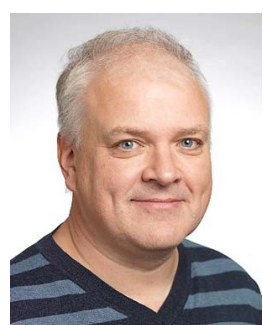

Thomas Eriksson received the Ph.D. degree in information theory from Chalmers University of Technology, Gothenburg, Sweden, in 1996. In 1997 and 1998, he was at AT\&T Labs Research in Murray Hill, NJ, USA, and in 1998 and 1999 he was at Ericsson Radio Systems AB, Kista, Sweden. Since 1999, he has been at Chalmers University, where he is a Professor in communication systems. Further, he was a Guest Professor at Yonsei University, $\mathrm{S}$. Korea, in 2003-2004. He is currently Vice Head of the Department of Signals and Systems at Chalmers, with responsibility for undergraduate and master education. His research interests include communication, data compression, and modeling and compensation of non-ideal hardware components (e.g., amplifiers, oscillators, modulators in communication transmitters and receivers).

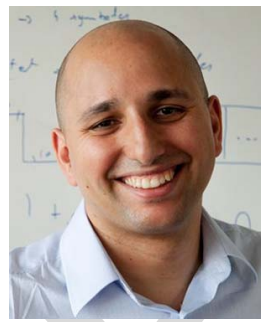

Mérouane Debbah (S'01-M'04-SM'08-F'15) received the M.Sc. and Ph.D. degrees from Ecole Normale Supérieure de Cachan, France, in 1996. He worked for Motorola Labs, Saclay, France, from 1999 to 2002, and the Vienna Research Center for Telecommunications (Vienna, Austria) until 2003. From 2003 to 2007, he joined the Mobile Communications Department of the Institut Eurecom (Sophia Antipolis, France) as an Assistant Professor. Since 2007, he has been a Full Professor at Supelec (Gifsur-Yvette, France). From 2007 to 2014, he was director of the Alcatel-Lucent Chair on Flexible Radio. Since 2014, he has been Vice-President of the Huawei France $\mathrm{R} * \mathrm{D}$ Center and Director of the Mathematical and Algorithmic Sciences Lab. His research interests are in information theory, signal processing, and wireless communications. He is an Associate Editor in Chief of the journal Random Matrix: Theory and Applications and was an Associate and Senior Area Editor for IEEE TRANSACTIONS ON SignAL PROCESSING respectively in 2011-2013 and 2013-2014. Dr. Debbah is a recipient of the ERC grant MORE (Advanced Mathematical Tools for Complex Network Engineering). He is a WWRF Fellow and a member of the academic senate of Paris-Saclay. He is the recipient of the Mario Boella award in 2005, the 2007 IEEE GLOBECOM Best Paper Award, the Wi-Opt 2009 Best Paper Award, the 2010 Newcom++ Best Paper Award, the WUN CogCom Best Paper 2012 and 2013 Award, the 2014 WCNC Best Paper Award as well as the Valuetools 2007, Valuetools 2008, CrownCom2009, Valuetools 2012 and SAM 2014 Best Student Paper Awards. In 2011, he received the IEEE Glavieux Prize Award and in 2012, the Qualcomm Innovation Prize Award. 


\title{
On Feedback Resource Allocation in Multiple-Input-Single-Output Systems Using Partial CSI Feedback
}

\author{
Behrooz Makki, Tommy Svensson, Senior Member, IEEE, Thomas Eriksson, and Mérouane Debbah, Fellow, IEEE
}

\begin{abstract}
This paper studies the problem of feedback resource allocation in multiple-input-single-output (MISO) channels utilizing partial channel state information (CSI) feedback. Considering low/moderate signal-to-noise ratios (SNRs), the optimal quantizers and the feedback bit allocation maximizing the throughput are obtained in the asymptotic case where the number of feedback bits increases. Moreover, the results are utilized to derive the optimal retransmission rates in the automatic repeat request (ARQ) protocols and joint CSI-ARQ schemes are proposed for the MISO setups. We show that uniform channel amplitude quantization is asymptotically optimal in terms of throughput. Also, the optimal retransmission rates of the incremental redundancy (INR) ARQ protocols follow an arithmetic progression in the exponential domain. Under certain conditions, a MISO system using quantized CSI can be mapped to a MISO or a SISO (S: single) setup using ARQ or joint CSI-ARQ feedback in the sense that they lead to the same throughput. Finally, to maximize the throughput, the optimal number of channel direction quantization bits should be $(M-1)$ times the number of amplitude quantization bits, where $M$ is the number of transmit antennas.
\end{abstract}

Index Terms-CSI quantization, HARQ feedback, MIMO transmission, throughput, joint phase-amplitude information feedback, incremental redundancy (INR) ARQ.

\section{INTRODUCTION}

D ESIGNING the optimal channel state information (CSI) quantization and feedback bit allocation for, e.g., maximizing the power-limited throughput is a complicated nonconvex problem which, depending on the fading model, may have no closed-form solution [1]-[3]. Particularly, the problem becomes more complex when the number of antennas at the transmitter(s) or receiver(s) increases. For this reason, most of the papers in the field of quantized CSI feedback focus on either channel amplitude quantization, e.g., [4], [5] or channel

Manuscript received May 30, 2014; revised September 25, 2014 and December 10, 2014; accepted January 22, 2015. This work was supported in part by the Swedish Governmental Agency for Innovation Systems (VINNOVA) within the VINN Excellence Center Chase. The editor coordinating the review of this paper and approving it for publication was M. C. Gursoy.

B. Makki, T. Svensson, and T. Eriksson are with the Department of Signals and Systems, Chalmers University of Technology, SE-412 96 Gothenburg, Sweden (e-mail: behrooz.makki@chalmers.se; tommy.svensson@ chalmers.se; thomase@chalmers.se).

M. Debbah is with Supelec, Plateau du Moulon, 91192 Gif-sur-Yvette, France (e-mail: merouane.debbah@supelec.fr).

Color versions of one or more of the figures in this paper are available online at http://ieeexplore.ieee.org.

Digital Object Identifier 10.1109/TCOMM.2015.2398861 direction quantization [6]-[15] and the quantization is normally based on random vector quantization or predefined tables of thresholds [13]-[16]. However, as also discussed in, e.g., [17][21], considerable performance improvement can be achieved when both partial channel direction and amplitude information are exploited for rate/power adaptation. On the other hand, feedback bit distribution is mostly based on simulations [22], [23] and the problem of joint CSI quantization and feedback bit allocation for maximizing the throughput has not been well formulated yet.

From another perspective, automatic repeat request (ARQ) is a sequential feedback approach to provide the transmitter with information about the channel quality. The optimization of the (re)transmission rates in the ARQ protocols is by itself a complex non-convex problem [2], [24]-[27]. However, as demonstrated in [2], [24] and in the following, under certain conditions the ARQ-based system can be mapped into an equivalent setup using quantized CSI feedback, in the sense that they lead to the same throughput. Thus, it is interesting to address the optimal CSI quantization and feedback bit distribution problem, because, as illustrated in the following, the results are useful not only for the quantized CSI schemes but also for deriving the optimal ARQ-based retransmission rates.

This paper studies the design of CSI quantization and feedback bit allocation in the multiple-input-single-output (MISO) setups. The optimization objective function is the system throughput. Also, the discussions are presented for the low/moderate signal-to-noise ratio (SNR) regimes and in the asymptotic conditions when the number of feedback bits increases. In the meantime, the analytical conclusions match with the numerical simulations with very high accuracy when tested for finite number of feedback bits. Moreover, we derive the optimal retransmission rates in the ARQ protocols, present joint CSI-ARQ approaches for the MISO networks and show conditions for when the quantized CSI, the ARQ and the joint CSI-ARQ feedback schemes can be mapped to each other.

The main conclusions of the paper are as follows:

- Considering low/moderate SNRs and product channel quantization codebooks with high number of feedback bits, uniform channel amplitude quantization is optimal in terms of throughput.

- The optimal low-SNR retransmission rates of the incremental redundancy (INR) ARQ protocols follow an arithmetic progression rule in the exponential domain when the number of retransmissions increases. 
- With proper setting of the number of quantization regions, the transmission power and the number of retransmissions, there exist mappings between the MISO and SISO (S: single) systems using joint channel direction and amplitude quantization, only channel amplitude quantization, the INR ARQ or joint CSI-INR ARQ feedback, in the sense that they result in the same throughput.

- With sufficiently large number of feedback bits and low/moderate SNRs, we have $\mathcal{Q}=(M-1) \mathcal{N}$ where $M$ is the number of transmit antennas and $\mathcal{Q}$ and $\mathcal{N}$ represent the optimal number of channel direction and amplitude feedback bits, respectively. Interestingly, our theoretical results are in harmony with the feedback bit allocation rules in, e.g., the 3GPP standards [1, Section IV.A].

Moreover, the numerical results indicate that the optimal channel amplitude quantization boundaries get closer to zero when the transmission power increases. Also, the efficiency of the uniform channel amplitude quantization is improved by increasing the number of transmit antennas. Finally, substantial throughput increment is achieved via very limited number of feedback bits, if the feedback signal is properly designed.

We should mention that the problem of CSI quantization has been previously studied in various papers, e.g., [2]-[21], but we develop new techniques and derive new conclusions that have not been presented before; as opposed to [2]-[16], we consider MISO setups, both the channel amplitude and direction quantizers are optimized in terms of throughput and we determine efficient feedback bit distribution between the channel direction and amplitude information maximizing the throughput. Moreover, the problem setup of the paper is different from the ones in [17]-[21] in which block diagnolization and predefined quantizers have been used for inter-cell interference suppression [17], numerical methods have been implemented to optimize (in terms of multiuser diversity gain) the number of quantization bits [18], a quantized CSI-based block diagonalization scheme is proposed for multiuser MIMO [19] and the feedback bit allocation has been optimized for outage-limited average power minimization [20], [21]. Finally, the discussions that we present about the ARQ and joint CSIARQ protocols have been considered by none of these papers.

\section{SySTEM MOdeL}

Consider a MISO system with $M$ transmit antennas which can be modeled as

$$
y=\sqrt{P} \mathbf{h}^{T} \mathbf{v} s+z .
$$

Here, $P$ is the transmission power, $\mathbf{h}=\left[h_{1} \ldots h_{M}\right]^{T} \in \mathcal{C}^{M}$ and $\mathbf{v}$ denote the fading random vector and the unit-norm beamforming vector, respectively, $s$ is the input signal and $z \in \operatorname{CN}(0,1)$ represents the white complex Gaussian noise added at the receiver. Also, $(.)^{T}$ denotes the transpose operator.

We study independent and identically distributed (iid) blockfading channels where the channel coefficients remain constant for a long time, determined by the channel coherence time, and then change according to their probability density functions (pdf:s). The results are obtained for the family of exponential distributions [28, Section 4.4] which can model most relevant fading conditions. Let $f_{G}$ and $F_{G}$ be the pdf and the cumulative distribution function (cdf) of a random variable in the family of exponential distributions. Among the properties of the family of exponential distributions are [28, Section 4.4]

$$
\begin{aligned}
& \forall g>0, \exists \mu_{1} \geq 0, \frac{\mathrm{d} f_{G}(g)}{\mathrm{d} g}<\mu_{1} \\
& \forall g>0, \exists \mu_{2} \geq 0, f_{G}(g)\left(\frac{\mathrm{d} f_{G}(g)}{\mathrm{d} g}\right)^{-1} \leq \mu_{2} .
\end{aligned}
$$

The receiver is assumed to have perfect CSI which is an acceptable assumption in block-fading channels [2]-[18], [20]. On the other hand, the transmitter is provided with quantized CSI (or ARQ feedback), as illustrated in the following. ${ }^{1}$ We concentrate on the continuous data communication model [2], [24]-[26] where a new codeword transmission starts as soon as the previous codeword transmission ends. Finally, the results are presented in natural logarithm basis and the throughput is given in nats-per-channel-use (npcu).

\section{Performance Analysis}

With the total of $B$ feedback bits, i.e., dividing $\mathrm{C}^{M}$ into $2^{B}$ quantization regions (QRs), the throughput of a MISO setup is obtained by

$$
\eta=\sum_{j=1}^{2^{B}} R_{j} \operatorname{Pr}\left(R_{j} \leq \log \left(1+P\left\|\mathbf{h}^{T} \mathbf{v}_{j}\right\|^{2}\right) \bigcap \mathbf{h} \in \xi_{j}\right)
$$

where $\mathbf{v}_{j}$ and $R_{j}$ denote the unit-norm beamforming vector and the data transmission rate considered for the $j$-th $\mathrm{QR}$, respectively, and $\xi_{j} \subset \mathrm{C}^{M}$ is the $j$-th $\mathrm{QR}$. Also, (4) is obtained by taking the expectation on the decodable data rates of all QRs.

To maximize the throughput, the boundaries of the QRs, the transmission rates $R_{j}$ and the beamforming vectors $\mathbf{v}_{j}$ should be optimized which, as stated before, cannot be determined with a closed-form solution. Therefore, suboptimal quantizers are normally considered and numerical solutions are utilized [4][18], [20], [21]. Here, we consider the class of product channel quantization codebooks which quantize the channel direction and amplitude information separately. Note that, while being suboptimal, the product quantization approach has some advantages such as lower storage requirements and faster quantization [20], [21], [29]. Also, many practical systems are equipped with channel amplitude quantizers [20]. Hence, the product quantization can be easily adopted in these systems.

With a product channel quantization approach, the channel amplitude and direction information are comprised as follows. Defining $G=\|\mathbf{h}\|^{2}$ as the squared channel amplitude, the distribution of the random variable $G$ is partitioned into $N$ QRs $\left[g_{n}, g_{n+1}\right)$, where $g_{n}$ 's are the amplitude quantization boundaries. Also, the channel direction information is quantized into $Q$ QRs with a uniform quantizer which is optimal for channel direction quantization in iid fading conditions. As examples, the 1- and the 2-dimensional representations of the QRs are

\footnotetext{
${ }^{1}$ The transmitter is assumed to know the long-term channel statistics, as it is required for parameter optimization.
} 


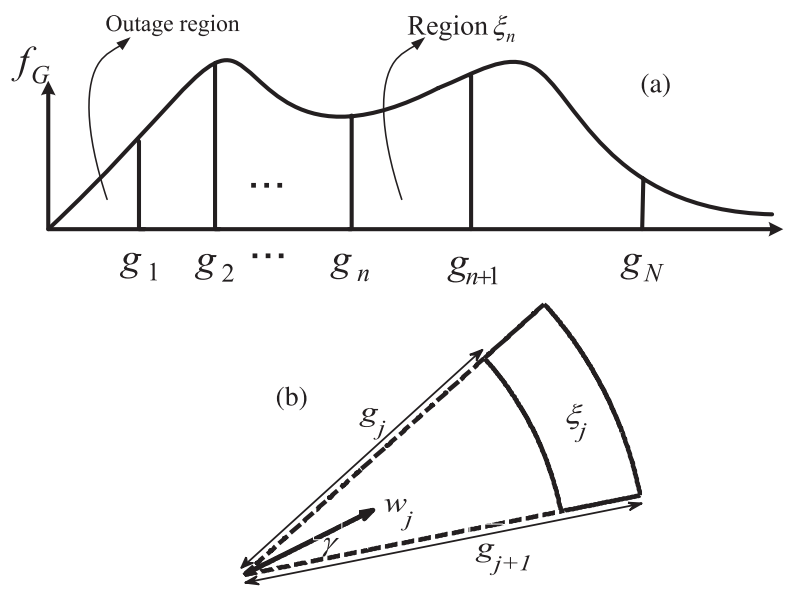

Fig. 1. The quantization boundaries in (a) a SISO and (b) a $2 \times 1$ MISO setup. With $M$ transmit antennas, the outage occurs iff the channel realization drops into the $M$-dimensional ball centered at the origin with radius $g_{1}$; otherwise the codeword is always decoded correctly.

represented in Fig. 1(a) and (b), respectively. Also, note that with the considered quantization scheme we have $N Q=2^{B}$.

Following the same procedure as in the SISO systems [2, Lemma 1], [3], it can be proved that the maximum throughput is achieved when the channel is assumed to be equal to its worst case in each QR. In this way, using (4), the maximum throughput is obtained by considering the beamformers according to

$$
\max _{\mathbf{v}_{j}} \inf _{\forall \mathbf{h} \in \xi_{j}}\left|\mathbf{h}^{T} \mathbf{v}_{j}\right|=\inf _{\forall \mathbf{h} \in \xi_{j}}\left|\mathbf{h}^{T} \mathbf{w}_{j}\right|=\sqrt{g_{j}} \cos \gamma
$$

where $\gamma$ is the angle of the QR (see Fig. 1(b) as an example). That is, the optimal beamformer in each QR is the unit-norm vector at the center of the region, denoted by $\mathbf{w}_{j}$, and the data transmission rate associated with the $j$-th $\mathrm{QR}$ is determined as

$$
R_{j}=\inf _{\forall \mathbf{h} \in \xi_{j}} \log \left(1+P\left|\mathbf{h}^{T} \mathbf{w}_{j}\right|^{2}\right)=\log \left(1+P g_{j} \cos ^{2} \gamma\right) .
$$

In this way, the outage occurs iff the channel realization drops into the $M$-dimensional ball centered at the origin with radius $g_{1}$; otherwise the codeword is always decoded correctly. Finally, due to the symmetry of the QRs in terms of direction parameters, the system throughput is found as

$$
\begin{aligned}
\eta & =\sum_{n=1}^{N} \log \left(1+P g_{n} \cos ^{2} \gamma\right) \operatorname{Pr}\left(G \in\left[g_{n}, g_{n+1}\right)\right) \\
& =\sum_{n=1}^{N} \log \left(1+P g_{n} \cos ^{2} \gamma\right)\left(F_{G}\left(g_{n+1}\right)-F_{G}\left(g_{n}\right)\right) .
\end{aligned}
$$

The objective of the paper is to maximize (7) subject to the total feedback budget $N Q=2^{B}$. Therefore, $N, Q$ and the quantization boundaries $g_{n}, \forall n$, should be properly designed. To achieve this goal, we consider the following procedure. First, we derive a closed-form relation between the quantization boundaries $g_{n}, \forall n$ (Lemma 1). The second step is to express the term $\cos ^{2} \gamma$, which represents the effect of quantized channel direction information, as a function of $Q$, i.e., the number of direction QRs. Here, we use the results of [11] leading to (13). Then, the results of Lemma 1 are utilized to write the quantization boundaries as a function of the number of amplitude QRs $N$. Lemmas 2 and 3 are dedicated to derive this function. Following the steps, (7) is rephrased as (17) which is a function of $N$ and $Q$. Therefore, adding the constraint $N Q=2^{B}$, we can determine the optimal feedback bit distribution rules, maximizing the throughput, as in (20). Indeed, to follow the steps, we need to use approximation and bounding techniques. In all bounding and approximation steps of the developed results, such as (12)-(14), and (19), we upper-bound the throughput. Also, we concentrate on the low-SNR regime with high resolution feedback, to keep the bounds/approximations reasonably tight. Moreover, as demonstrated in the following, the final conclusions are in harmony with the numerical simulations with high accuracy.

Let us first find a relationship between the quantization boundaries $g_{n}, \forall n$, as stated in Lemma 1 .

Lemma 1: With a product channel quantizer and low SNRs, the optimal, in terms of throughput, channel amplitude quantization tends towards uniform quantization, when the number of feedback bits increases asymptotically.

Proof: Setting $\frac{\partial \eta}{\partial g_{n}}=0$ in (7) leads to

$\frac{P \cos ^{2} \gamma\left(F_{G}\left(g_{n+1}\right)-F_{G}\left(g_{n}\right)\right)}{1+P \cos ^{2} \gamma g_{n}}+f_{G}\left(g_{n}\right) \log \left(\frac{1+P \cos ^{2} \gamma g_{n-1}}{1+P \cos ^{2} \gamma g_{n}}\right)=0$.

Assuming low $\mathrm{SNR}$ regimes, i.e., $P \rightarrow 0$, (8) is rephrased as

$$
F_{G}\left(g_{n+1}\right)-F_{G}\left(g_{n}\right)+f_{G}\left(g_{n}\right)\left(g_{n-1}-g_{n}\right)=0
$$

which leads to

$$
g_{n}=\frac{g_{n+1}+g_{n-1}}{2} .
$$

Here, we have used $\log (1+t) \rightarrow t$ for small values of $t$ and (10) follows from $\left(F_{G}\left(g_{n+1}\right)-F_{G}\left(g_{n}\right)\right) \rightarrow f_{G}\left(g_{n}\right)\left(g_{n+1}-g_{n}\right)$ for sufficiently large number of QRs. Thus, (10) indicates that, at low SNRs, the quantization boundaries follow an arithmetic progression. That is, the optimal, in terms of throughput, channel amplitude quantization tends towards uniform quantization, when the number of QRs increases.

Interestingly, the conclusion of the lemma is independent of the fading pdf and the transmission power as long as we are in low SNR regime and the number of QRs is high. Also, with uniform channel quantization we have

$$
g_{n}=g_{1}+(n-1) \alpha
$$

where $\alpha$ is the step size of the uniform quantization. Here, it is interesting to note that, although Lemma 1 proves the asymptotic optimality of the uniform quantizers in high-resolution feedback regimes, we still need to find the proper step size such that the entire range of positive values is covered by the quantizer and the gradient of the throughput, with respect to the quantization boundaries, goes to zero. The optimality condition of the step size is derived in Lemma 3 as explained in the following.

The following discussions are presented for the low SNR regime where, using (7), the normalized throughput function (normalized by the transmission power $P$ ) is given by

$$
\tilde{\eta}=\cos ^{2} \gamma \sum_{j=1}^{N} g_{j}\left(F_{G}\left(g_{j+1}\right)-F_{G}\left(g_{j}\right)\right) .
$$


This is an appropriate approximation at low SNRs (see [30] and the references therein). Also, note that the low-SNR regime is of interest in many communication scenarios such as wideband systems, sensor networks, cognitive radio, deep-space communications via satellites and communication at the edge of the cellular networks, e.g., [31], [32], where the available power per degree of freedom can be vanishingly small.

To follow the steps and rewrite (12) as a function of $Q$ and $N$, we use the bounds obtained in [11, eq. 15], i.e.,

$$
\begin{aligned}
(\sin \gamma)^{-2(M-1)}< & Q<\left(\frac{\sin \gamma}{2}\right)^{-2(M-1)} \\
& \Rightarrow 1-4 Q^{-\frac{1}{M-1}}<\cos ^{2} \gamma<1-Q^{-\frac{1}{M-1}}
\end{aligned}
$$

to upper bound the direction-related part of (12) as $\cos ^{2} \gamma<$ $1-Q^{-\frac{1}{M-1}}$. Then, Lemma 2 is utilized to upper bound the summation part of (12) which reflects the effect of channel amplitude quantization.

Lemma 2: The normalized throughput (12) is upper bounded by

$$
\begin{aligned}
& \frac{\tilde{\eta}}{\mathrm{E}\{G\} \cos ^{2} \gamma} \leq 1+\frac{\alpha(N-1)}{g_{1}} \\
&-\sum_{j=1}^{\infty}\left(\frac{-\alpha}{g_{1}}\right)^{j+1}\left\{\frac{1}{j+1} \sum_{k=0}^{j}\left(\begin{array}{c}
j+1 \\
k
\end{array}\right) B_{k}(N-1)^{j+1-k}\right\}
\end{aligned}
$$

where $B_{k}$ 's are the Bernoulli numbers, $\mathrm{E}\{$.$\} is the expectation$ operator and $\left(\begin{array}{l}n \\ k\end{array}\right)$ denotes the " $n$ choose $k$ " operator.

Proof: Using (12), the bound is obtained by

$$
\begin{aligned}
\frac{\tilde{\eta}}{\cos ^{2} \gamma} \stackrel{(a)}{=} g_{1} \operatorname{Pr}\left(G \geq g_{1}\right)+\alpha \sum_{n=2}^{N} \operatorname{Pr}\left(G \geq g_{n}\right) \\
\stackrel{(b)}{\leq} \mathrm{E}\{G\}\left(1+\sum_{n=2}^{N} \frac{\alpha}{g_{n}}\right) \\
\stackrel{(c)}{=} \mathrm{E}\{G\}\left(1+\frac{\alpha}{g_{1}} \sum_{n=1}^{N-1}\left(1+\sum_{j=1}^{\infty}(-1)^{j}\left(\frac{n \alpha}{g_{1}}\right)^{j}\right)\right) \\
=\mathrm{E}\{G\}\left(1+\frac{\alpha(N-1)}{g_{1}}-\sum_{j=1}^{\infty}\left(\frac{\alpha}{g_{1}}\right)^{j+1}\right. \\
\left.\times\left\{\frac{1}{j+1} \sum_{k=0}^{j}\left(\begin{array}{c}
j+1 \\
k
\end{array}\right) B_{k}(N-1)^{j+1-k}\right\}\right) .
\end{aligned}
$$

Here, $(a)$ comes from (11) and some manipulations and $(b)$ is based on Markov's inequality $\operatorname{Pr}(G \geq x) \leq \frac{\mathrm{E}\{G\}}{x}, x \geq 0[33$, p. 91]. Then, $(c)$ is obtained by (11) and Taylor expansion of the function $\frac{1}{1+x}$ and the last equality follows from some manipulations and the definition of Bernoulli numbers [34].

Lemma 2 rephrases the throughput as a function of the first quantization boundary $g_{1}$ and $\alpha$. Therefore, the next step to write (7) based on $Q$ and $N$ is to represent $\alpha$ as a function of $N$. The representation is derived in Lemma 3 as follows.
Lemma 3: With sufficiently high number of QRs and low SNRs, the optimal, in terms of throughput, quantization step size $\alpha$ is given by

$$
\alpha=N^{-\varepsilon(N)}, \lim _{N \rightarrow \infty} \varepsilon(N) \rightarrow 1
$$

where $\varepsilon(N)$ can be any function of the total number of QRs $N$.

Proof: The proof follows from the fact that, for every given power, a necessary condition for the optimality of the quantization approach is that the gradient of the throughput function with respect to the quantization boundaries goes to zero. Thus, (16) is obtained by finding the gradient of (12) with respect to $g_{n}$ 's, and letting it go to zero. More details can be found in the Appendix. ${ }^{2}$

Using (13), (14), and (16), the normalized throughput (12) is represented as

$$
\tilde{\eta} \leq \mathrm{E}\{G\}\left(1-Q^{-\frac{1}{M-1}}\right)
$$

$$
\begin{gathered}
\times\left(1+\frac{N^{-\varepsilon(N)}}{g_{1}}(N-1)-\sum_{j=1}^{\infty}\left(-\frac{N^{-\varepsilon(N)}}{g_{1}}\right)^{j+1}\right. \\
\left.\times\left\{\frac{1}{j+1} \sum_{k=0}^{j}\left(\begin{array}{c}
j+1 \\
k
\end{array}\right) B_{k}(N-1)^{j+1-k}\right\}\right)
\end{gathered}
$$

which is a function of $N$ and $Q$; this was our ultimate goal. Then, with a feedback budget constraint $N Q=2^{B}$, (17) is used to formulate the optimal, in terms of throughput, feedback bit distribution problem as

$$
\begin{aligned}
\max _{N, Q}\left\{( 1 - Q ^ { - \frac { 1 } { M - 1 } } ) \left(1+\frac{N^{-\varepsilon(N)}}{g_{1}}(N-1)-\sum_{j=1}^{\infty}\left(-\frac{N^{-\varepsilon(N)}}{g_{1}}\right)^{j+1}\right.\right. \\
\left.\left.\quad \times\left\{\frac{1}{j+1} \sum_{k=0}^{j}\left(\begin{array}{c}
j+1 \\
k
\end{array}\right) B_{k}(N-1)^{j+1-k}\right\}\right)\right\} \\
\text { s.t. } N Q=2^{B} .
\end{aligned}
$$

Using $B_{0}=1, B_{1}=\frac{1}{2}, \lim _{N \rightarrow \infty} \varepsilon(N) \rightarrow 1$ and the first-order approximation of the summation term in (17) by removing the terms $N^{-i}, i>1$, for large values of $N$, we take the logarithm of the objective function to rewrite (18) as

$$
\begin{aligned}
& \max _{N, Q}\left\{\log \left(1-\frac{1}{a N}\right)+\log \left(1-Q^{-\frac{1}{M-1}}\right)\right\} \\
& \qquad \stackrel{(d)}{=} \min _{N, Q}\left\{\frac{1}{a} N^{-1}+Q^{-\frac{1}{M-1}}\right\} \\
& \text { s.t. } N Q=2^{B}
\end{aligned}
$$

\footnotetext{
${ }^{2}$ Along with Lemma 3 , it is interesting to note that, with the family of exponential distributions and the optimal design of the high-resolution uniform quantizer, the expected overload distortion (related to the outer region of the quantizer) tends to zero faster than the granular distortion (corresponding to the in-region distortion) [35].
} 
where $a \doteq \frac{1+\frac{1}{g_{1}}-\sum_{j=1}^{\infty} \frac{1}{j+1}\left(\frac{-1}{g_{1}}\right)^{j+1}}{\frac{1}{g_{1}}-\frac{1}{2} \sum_{j=1}^{\infty}\left(\frac{-1}{g_{1}}\right)^{j+1}}$ and $(d)$ holds for large values of $N$ and $Q$ on which we focus. The optimal values of $N$ and $Q$ are obtained by setting $\frac{1}{a} N^{-1}=Q^{-\frac{1}{M-1}}, N Q=2^{B}$, which defining $Q \doteq \log _{2}(Q), \mathcal{N} \doteq \log _{2}(N)$ and $\mathcal{A} \doteq \log _{2}(a)$ leads to

$$
\begin{aligned}
\mathcal{Q} & =\frac{M-1}{M} B+\frac{M-1}{M} \mathcal{A}, \\
\mathcal{N} & =\frac{1}{M} B-\frac{M-1}{M} \mathcal{A} .
\end{aligned}
$$

The optimal numbers of bits are the closest integers to (20), (21). Interestingly, for large values of $B,(20),(21)$ result in

$$
\mathcal{Q}=(M-1) \mathcal{N} .
$$

That is, for sufficiently large number of feedback bits and low/moderate SNRs, the optimal number of quantization bits considered for channel direction information feedback tends to be $(M-1)$ times the number of feedback bits required for channel amplitude information feedback.

Regarding the derived bit allocation rule, the following points worth to be mentioned:

- The derived bit allocation rule is independent of the fading pdf and the transmission power as long as the transmission power is sufficiently low. Also, (22) holds for different step sizes as long as the optimality condition of Lemma 3 is satisfied.

- With a different amplitude quantization approach, the same bit allocation rule as in (22) has been previously derived by [20] for a different problem where the goal has been to design the power controllers for the MISO setups in outage-limited conditions. There, the optimal amplitude QRs minimizing the outage-limited transmission power have been shown to follow a geometric sequence, as opposed to our results with uniform channel amplitude quantization.

- Our theoretical conclusion is in line with, e.g., the standard 3GPP Release-99 where, with 2 transmit antennas, 2 and 3 feedback bits are considered for channel amplitude and direction quantization, respectively [1, Section IV.A] (With $M=2$ and $B=5,(20)$, (21) are rephrased as $Q=$ $2.5+0.5 \mathcal{A}, \mathcal{N}=2.5-0.5 \mathcal{A}$ which for the practical range of $\mathcal{A}$ lead to the integer numbers of bits $Q=3, \mathcal{N}=2$ ).

- We presented the analytical results for iid fading conditions, in harmony with [4], [5], [9]-[18], [20]. Limited feedback in correlated Rayleigh-fading MISO setups has been previously studied in, e.g., [6]-[8]. Particularly, as shown in [7], at low/moderate SNRs and with highresolution feedback, on which we focus, the capacity loss/decaying factor of the iid and correlated Rayleighfading channels, due to channel quantization, are the same and are given by [7, eq. (43)-(45)] which correspond to (13). Therefore, although we present the results for iid channels, (with high accuracy) the same bit allocation rule as in (22) holds for the correlated channels at low/moderate SNRs as well.

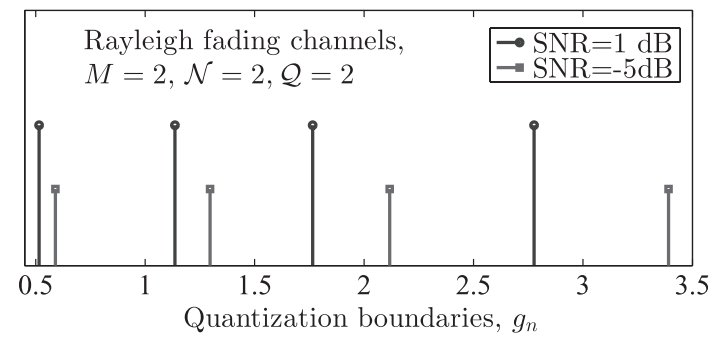

Fig. 2. The optimal quantization boundaries maximizing the throughput, Rayleigh-fading channel, $M=2, \mathcal{Q}=\mathcal{N}=2$. As demonstrated in the following, the results can be mapped to the optimal retransmission rates in a MISO setup utilizing joint CSI-INR ARQ with $Q$ channel direction feedback bits and a maximum of $I+1=2^{\mathcal{N}}$ INR-based retransmissions. Also, with proper scaling of the transmission SNR, the results of the figure give (1) the optimal retransmission rates in a MISO-INR ARQ setup utilizing perfect channel direction information and (2) the optimal QRs in a MISO setup with quantized channel amplitude and perfect channel direction information. See Theorem 1 and its following discussions for more details.

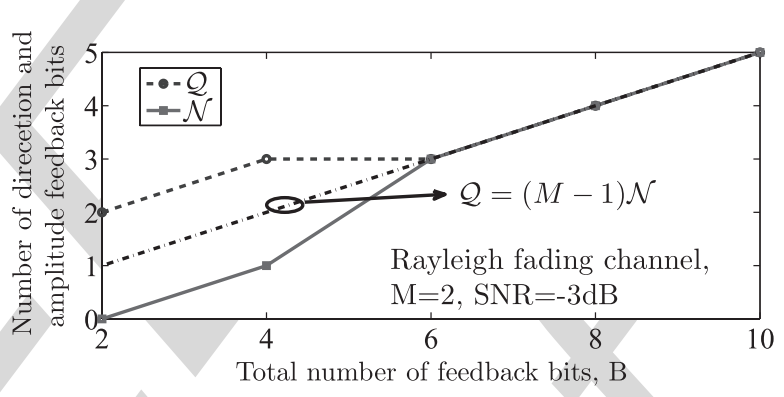

Fig. 3. The optimal, in terms of throughput, number of channel amplitude and direction feedback bits versus the total number of feedback bits $B$, Rayleighfading channel, $M=2, \mathrm{SNR}=-3 \mathrm{~dB}$. The results also give the optimal feedback resource allocation between the channel direction quantization and INR ARQ feedback in a joint CSI-INR ARQ feedback approach. See Theorem 1 and its following discussions for more details.

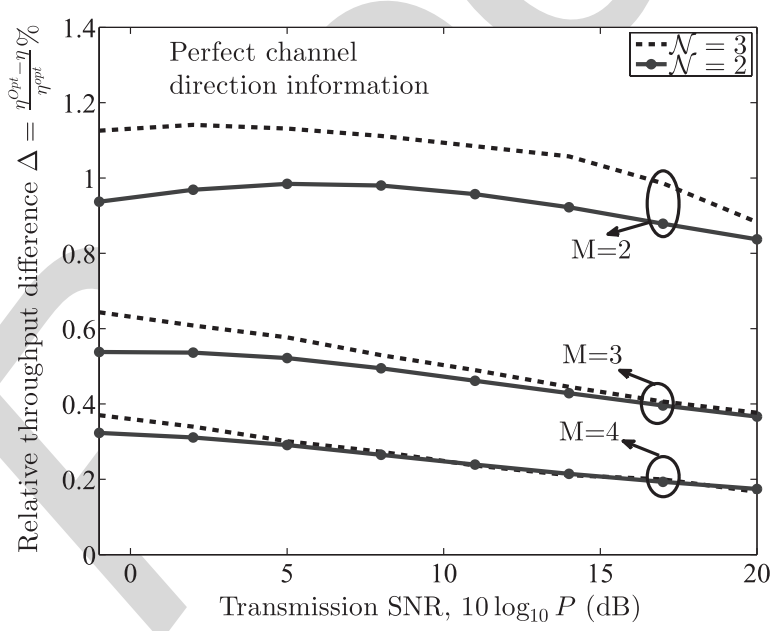

Fig. 4. The relative throughput difference $\Delta=\frac{\eta^{\text {opt }}-\eta}{\eta^{\text {opt }}} \%$ for different numbers of transmit antenna $M$, Rayleigh-fading channel. The results are obtained with perfect channel direction information at the transmitter, while they can be mapped to the cases with quantized channel direction feedback and scaled transmission SNRs (see Theorem 1).

- Our results are derived based on the high-resolution feedback assumption. However, as a well-known result in the source coding area, the high-resolution bounds agree well with the simulation results when tested for low resolutions, e.g., [7], [35] (see Figs. 2-6 as well). 


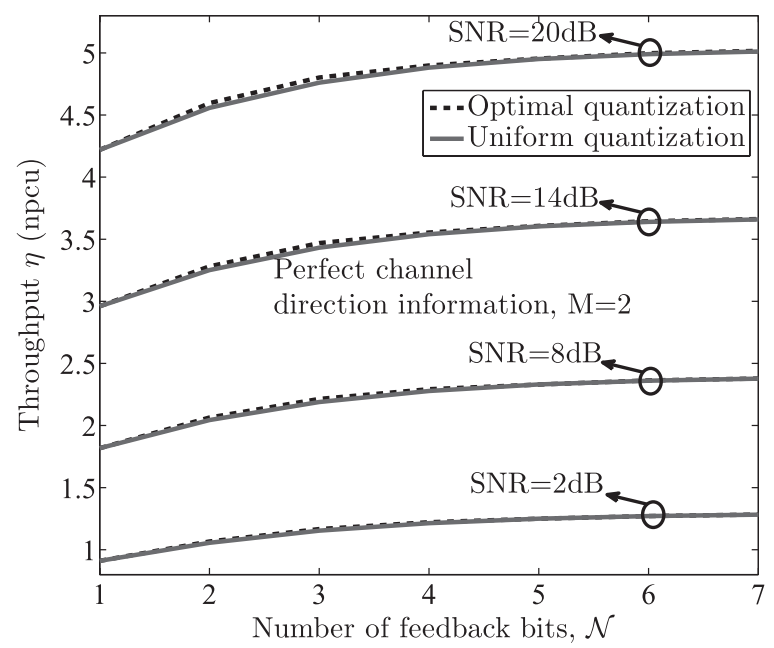

Fig. 5. The throughput for different transmit SNRs and numbers of amplitude feedback bits $\mathcal{N}$. Rayleigh-fading channel, $M=2$. The results are obtained with perfect channel direction information at the transmitter, while they can be mapped to the cases with quantized channel direction feedback and scaled transmission SNRs (see Theorem 1).

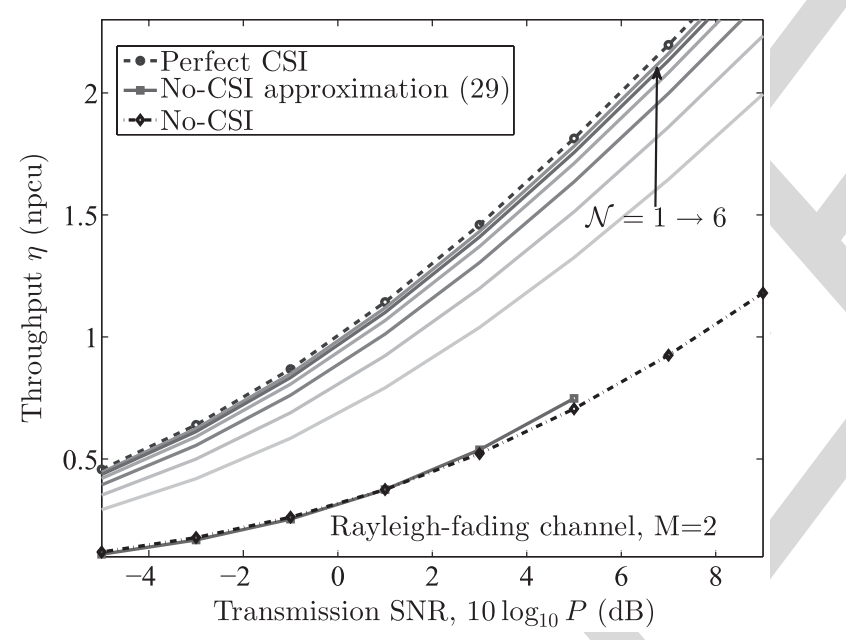

Fig. 6. The throughput in a Rayleigh-fading MISO channel with different levels of CSI feedback. The same throughput as in the cases with $\mathcal{N}$ channel amplitude feedback bits and perfect channel direction feedback is achieved when utilizing (1) both channel amplitude and direction quantization, (2) joint quantized channel direction information and INR ARQ feedback, and (3) only INR ARQ feedback, if the number of quantization bits, the transmission power and the number of ARQ retransmission rounds are adapted according to Theorem 1.

In Figs. 2-6, we evaluate the simulation results for Rayleighfading channels where each element of the channel vector $\mathbf{h}$ follows $\operatorname{eN}(0,1)$. Demonstrated in Fig. 2 are the optimal channel amplitude quantization boundaries for different transmission SNRs $10 \log _{10} P$ and $\mathcal{N}=\mathcal{Q}=2$. Also, setting $M=2$ and SNR $=-3 \mathrm{~dB}$, Fig. 3 shows the optimal bit distribution obtained by exhaustive search and compares the results with the ones obtained via our theoretical derivation (22). Here, the results are obtained for the optimal and uniform quantization approaches which, for the considered parameter setting of the figure, lead to the same optimal bit allocations demonstrated in the figure. The effect of different channel quantization schemes on the throughput is investigated in Figs. 4 and 5. Here, for different numbers of transmit antennas, the relative throughput difference function $\Delta=\frac{\eta^{\text {opt }}-\eta}{\eta^{\text {opt }}} \%$ is plotted where $\eta$ denotes the throughput achieved with uniform channel amplitude quantization and $\eta^{\text {opt }}$ is the maximum throughput achieved by exhaustive search on the amplitude QRs (Fig. 4). Also, considering perfect channel direction information, Fig. 5 shows the throughput for different quantization schemes and $M=2$. The results are obtained for different total numbers of feedback bits and transmission SNRs.

As demonstrated in the figures, the optimal channel amplitude quantization is very close to uniform quantization even for limited number of feedback bits (Fig. 2). However, the last quantization boundary of the optimal quantizer grows large, as expected. Moreover, the optimal channel amplitude quantization boundaries get closer to zero when the transmission power increases (Fig. 2). The theoretical results of (20)-(22) are in harmony with the optimal feedback bit allocation rules derived via simulations, when the total number of feedback bits increases (Fig. 3). Also, it is interesting to note that optimizing the throughput at high SNRs we observe the optimal number of channel direction quantization bits to be higher (or equal) than the ones allocated for amplitude quantization. However, depending on the number of antennas and the SNR, different optimal bit allocations are obtained at high SNRs; this is expected because our results have not been proved for high SNRs. In Fig. 4, we observe higher relative throughput difference in the cases with $\mathcal{N}=3$, compared to the case of $\mathcal{N}=2$, which is because of the finite number of feedback bits and the range of SNR in the figure. On the other hand, the efficiency of the uniform channel amplitude quantization is improved by increasing the number of transmit antennas/feedback bits, and the difference between the throughput of the optimal and uniform quantization-based schemes is negligible even with limited number of feedback bits (Figs. 4 and 5). Particularly, for a large range of SNRs the relative throughput difference is less than $1 \%$ (Fig. 4).

To close the discussions, we should mention that throughout the paper we concentrate on the single-user MISO setups. Our reasons for selecting the MISO systems are 1) different standards such as the $3 \mathrm{GPP}[1$, Section IV.A] and the $3 \mathrm{GPP}$ LTE [1, Section IV.D], [36] mainly concentrate on the limited feedback schemes for the MISO setups, 2) the analytical results can be derived/followed, 3) a large portion of the related literature is devoted to the MISO systems, e.g., [6]-[8], [10], [14], [20], [36]. However, our results also provide some insights/tools for the cases with multiuser MIMO. An interesting extension of the paper, in which we are currently involved, is to study the feedback distribution in multiuser systems. Intuitively, we expect the same conclusions as in the single-user MISO setup, when we extend the results to the multiuser systems. Particularly, considering the outage-limited power minimization problem in the multiuser setups, [21] shows the same feedback bit distribution rule as in (22). Finally, deriving feedback bit distribution rules based on the lower bounds of the throughput is an interesting topic for extensions. 


\section{A. Relevance to the ARQ-Based Schemes}

It has been previously demonstrated that the throughput of a system utilizing INR ARQ protocol with a maximum of $I+1$ (re)transmission rounds, continuous data communication and uniform power allocation is obtained by [2], [24] ${ }^{3}$

$$
\eta^{\mathrm{INR}}=\sum_{i=1}^{I+1} R_{i}^{\mathrm{INR}}\left(F_{G}\left(\frac{e^{R_{i-1}^{\mathrm{INR}}}-1}{P}\right)-F_{G}\left(\frac{e^{R_{i}^{\mathrm{INR}}}-1}{P}\right)\right) .
$$

Here, $R_{i}^{\mathrm{INR}}, R_{0}^{\mathrm{INR}} \doteq \infty$, is the equivalent data rate at the end of the $i$-th round. Then, with a SISO setup, [2, Theorem 1], [24, Lemma 3] have used (23) to relate the throughput of the ARQ-based scheme to the throughput in the quantized CSI-based approach. The following theorem extends the results of [2], [24] to a MISO setup. Moreover, we derive the optimal, in terms of throughput, rate allocation of the INR ARQ and propose joint CSI-ARQ protocols which lead to the same throughput as in the cases with only CSI quantization. The theorem is of interest because 1) it uses the results of the quantized CSI scheme to solve the problem of optimal retransmission rate allocation in the MISO-INR systems; the problem which is complex non-convex in general. Moreover, 2) there are many papers using only channel amplitude or channel direction quantization. Then, the theorem provides connections between the papers considering one of these schemes.

Theorem 1: The following assertions are valid:

a) Maximizing the low-SNR throughput with asymptotically high number of retransmissions, the equivalent data rates of the INR protocol follow an arithmetic progression in the exponential domain.

b) In the optimal case, the same throughput is achieved in the following scenarios:

- Scenario 1: A MISO setup with product CSI quantization consisting of $N$ and $Q$ QRs for the channel amplitude and direction quantization, respectively, and transmission power $P$.

- Scenario 2: A MISO setup utilizing joint CSI-ARQ feedback as follows. First, a uniform channel direction quantization with $Q$ QRs is used to inform the transmitter about the channel direction. Then, with no pre-knowledge about the channel amplitude, the data is transmitted via an INR ARQ with a maximum of $I+1=N$ retransmissions and power $P$.

- Scenario 3: A MISO-INR approach with a maximum of $I+1=N$ retransmissions, perfect channel direction information and transmission power $P \cos ^{2} \gamma$ with $\gamma$ obtained in Scenario 1.

- Scenario 4: A MISO setup with $N$ channel amplitude QRs, perfect channel direction information and transmission power $P \cos ^{2} \gamma$ with $\gamma$ obtained in Scenario 1.

In all considered scenarios, the throughput is given by (7).

${ }^{3}$ In [2], [24], the SISO model is considered. But, the same argument as in [2], [24] is valid for the MISO setup too.
Proof: Using the variable transformation $R_{I+2-i}^{\mathrm{INR}} \dot{=}$ $\log \left(1+P g_{i}\right)$ and replacing $P \cos ^{2} \gamma$ by $P,(23)$ is mapped to (7) with $N=I+1$. Therefore, following the same arguments as in Lemma 1, we have

$$
e^{R_{i}^{\mathrm{INR}}}=\frac{e^{R_{i+1}^{\mathrm{INR}}}+e^{R_{i-1}^{\mathrm{INR}}}}{2}
$$

in the optimal case, which follows from (10) and $R_{I+2-i}^{\mathrm{INR}} \dot{=}$ $\log \left(1+P g_{i}\right)$. That is, at low SNRs, the equivalent data rates of the INR protocol follow an arithmetic progression in the exponential domain, as stated in part (a) of the theorem.

For part (b), the proof follows from (7) and (23) where, using the variable transform $R_{I+2-i}^{\mathrm{INR}} \doteq \log \left(1+P g_{i}\right)$ and replacing $P \cos ^{2} \gamma$ by $P$ whenever required, it can be shown that the same throughput is achieved in all considered scenarios. Note that, in contrast to part (a), there is no approximation in part (b) and the conclusion holds for every transmission power/number of QRs.

We should mention that the result of Theorem 1 part (a) is different from the one in [25, Theorem 1] which studies the performance of INR ARQ protocols in the bursty data communication models. There, the optimal retransmission rates have been shown to follow a geometric sequence $R_{i}^{\mathrm{INR}}=$ $\sqrt{R_{i-1}^{\mathrm{INR}} R_{i+1}^{\mathrm{INR}}}$, as opposed to the conclusion of Theorem 1 part (a) with $e^{R_{i}^{\mathrm{INR}}}=\frac{e_{i+1}^{R_{i+1}^{\mathrm{NR}}}+e_{i-1}^{R_{i-1}}}{2}$. As discussed in [24], [25], there are fundamental differences between the performance of ARQ protocols in the bursty and continuous data communication models. Specially, the ARQ-based throughput achieved in these data communication models are different which results in differences in the optimal retransmission rates.

Following the arguments of Theorem 1, (22) gives the optimal feedback resource allocation between the channel direction quantization feedback bits and the INR-based ARQ feedback bits in the joint CSI-ARQ model of Theorem 1 (see Fig. 3). Moreover, using $R_{I+2-i}^{\mathrm{INR}} \doteq \log \left(1+P g_{i}\right)$, Fig. 2 gives the optimal INR-based retransmission rates of the considered joint CSIARQ approach with $Q=2$. Also, with proper scaling of the transmission SNR, the results of the figure give 1) the optimal retransmission rates in a MISO-INR ARQ setup utilizing perfect channel direction information and 2) the optimal quantization boundaries in a MISO setup with quantized channel amplitude and perfect channel direction information.

Theorem 1 presented equivalent models for different MISO setups, while there are also mappings between the MISO and SISO systems utilizing partial CSI feedback. For instance, the throughput achieved in a MISO setup with $N$ and $Q$ channel amplitude and direction QRs, respectively, is the same as the throughput achieved in a SISO system with $N$ channel amplitude QRs, transmission power $P \cos ^{2} \gamma$ and channel gain cdf $F_{G}$. This is because in both cases the throughput is given by (7). Finally, Theorem 1 proposed different joint CSI-ARQ, ARQ, quantized CSI with channel direction and amplitude (or each one alone) feedback models which lead to the same throughput. Then, selecting the best scheme depends on many parameters such as the implementation complexity, delay requirement and other protocol aspects. 
Fig. 6 demonstrates the system throughput in a $2 \times 1$ Rayleigh-fading MISO setup. Here, considering $\mathcal{N}=1, \ldots, 6$ number of amplitude feedback bits, the throughput is obtained with uniform channel amplitude quantization, and the results are compared with cases having perfect/no CSI feedback. Again, we can follow the discussions of Theorem 1 to map the results to the cases with different quantized CSI, ARQ or joint CSI-ARQ feedback models. Also, the difference between the throughput of the optimal and uniform quantizationbased schemes is negligible for the considered range of SNR. Thus, we only plot the results of the uniform quantizationbased scheme. Note that for Rayleigh fading conditions we have $f_{G}(x)=\frac{1}{\Gamma(M)} x^{M-1} e^{-x}$ and $F_{G}(x)=1-\frac{\Gamma(M, x)}{\Gamma(M)}, x \geq 0$, with $\Gamma(x)$ and $\Gamma(m, x)$ representing the Gamma and the incomplete Gamma functions, respectively. Therefore, the perfectCSI throughput presented in Fig. 6 is obtained by

$$
\begin{aligned}
\eta^{\text {perfect }} & =\int_{0}^{\infty} \frac{1}{\Gamma(M)} x^{M-1} e^{-x} \log (1+P x) \mathrm{d} x \\
& \stackrel{(e)}{=} \frac{1}{\Gamma(M)} \sum_{j=1}^{\infty} \frac{(-1)^{j+1} P^{j}}{j} \int_{0}^{\infty} e^{-x} x^{M+j-1} \mathrm{~d} x \\
& =\frac{1}{\Gamma(M)} \sum_{j=1}^{\infty} \frac{(-1)^{j} P^{j}}{j} \Gamma(M+j)
\end{aligned}
$$

where $(e)$ comes from the Taylor expansion of $\log (1+P x)$. Also, the no-CSI throughput is given by

$$
\begin{aligned}
\eta^{\mathrm{No}} & =\max _{R}\left\{R\left(1-F_{G}\left(\frac{M\left(e^{R}-1\right)}{P}\right)\right)\right\} \\
& =\max _{x}\left\{\log \left(1+\frac{P}{M} x\right)\left(1-F_{G}(x)\right)\right\} \\
& =\frac{1}{\Gamma(M)} \max _{x}\left\{\log \left(1+\frac{P}{M} x\right) \Gamma(M, x)\right\}
\end{aligned}
$$

which, depending on the value of $M$, may have no closedform solution. ${ }^{4}$ However, as the first-order approximation, we can use $\log \left(1+\frac{P}{M} x\right) \approx \frac{P}{M} x$ and $\Gamma(M, x) \approx(M-1) !-\frac{x^{M}}{M}$ to rephrase the maximization problem of (26) as

$$
\begin{aligned}
\frac{1}{\Gamma(M)} \max _{x}\left\{\log \left(1+\frac{P}{M} x\right) \Gamma(M, x)\right\} & \\
& \approx \frac{P}{M^{2} \Gamma(M)} \max _{x}\left\{x\left(M !-x^{M}\right)\right\}
\end{aligned}
$$

and find a low-SNR approximation of the no-CSI throughput as

$$
\eta^{\text {No }} \approx \log \left(1+\frac{P}{M} \sqrt[M]{\frac{M !}{M+1}}\right) \frac{\Gamma\left(M, \sqrt[M]{\frac{M !}{M+1}}\right)}{\Gamma(M)} .
$$

\footnotetext{
${ }^{4}$ Setting $M=1$ as a special case, the no-CSI throughput of a SISO setup is given by

$$
\eta^{\mathrm{No}}=\max _{x}\left\{e^{-x} \log (1+x P)\right\}=\Lambda(P) e^{-\frac{e^{\Lambda(P)}-1}{P}}
$$

where $\Lambda($.) denotes the Lambert $\mathrm{W}$ function.
As shown in Fig. 6, the approximation (29) is tight at low SNRs and the tightness decreases with the SNR. Also, considering different transmission SNRs, substantial throughput increment is observed with limited number of amplitude QRs.

\section{CONCLUSION}

This paper studied the design of optimal CSI quantization and feedback bit allocation in MISO systems when the number of feedback bits increases. Also, we presented discussions on how to select the (re)transmission rates of the INR ARQ protocol such that the throughput is maximized. In terms of throughput, the results showed that: 1) uniform quantization is asymptotically optimal for MISO channels. 2) The INR-based retransmission rates follow an arithmetic progression in the exponential domain. 3) For sufficiently large number of feedback bits, the optimal number of channel direction quantization bits tends to be $(M-1)$ times the number of channel amplitude quantization bits where $M$ is the number of transmit antennas. Finally, 4) there exist mappings between the throughput of the MISO systems utilizing INR ARQ, joint CSI-ARQ or different quantized CSI schemes.

\section{APPENDIX}

\section{A. Proof of Lemma 3}

Using (12) with uniform amplitude quantization, we derive the gradient of the throughput as

$$
\begin{gathered}
\left\{\begin{aligned}
\left|\frac{\partial \tilde{\eta}}{\partial g_{n}}\right| & =\left|\int_{g_{n}}^{g_{n+1}} f_{G}(t) \mathrm{d} t-f_{G}\left(g_{n}\right)\left(g_{n-1}-g_{n}\right)\right| \\
& \stackrel{(f)}{\leq} \frac{1}{2} \max _{x \in\left[g_{n}, g_{n+1}\right]}\left(\frac{\mathrm{d} f_{G}(x)}{\mathrm{d} x}\right) \alpha^{2} \\
\left|\frac{\partial \tilde{\eta}}{\partial g_{N}}\right| & =\left|f_{G}\left(g_{N}\right)\left(g_{n-1}-g_{N}\right)+1-f_{G}\left(g_{N}\right)\right| \\
& =\left|1-f_{G}\left(g_{N}\right)-\alpha f_{G}\left(g_{N}\right)\right| \doteq \Phi
\end{aligned}\right. \\
\begin{aligned}
\|\nabla\{\tilde{\eta}\}\| & =\left(\sum_{\forall g_{n}}\left|\frac{\partial \tilde{\eta}}{\partial g_{n}}\right|^{2}\right)^{\frac{1}{2}} \leq \Phi+\left(\sum_{\forall g_{n}, n \neq N}\left|\frac{\partial \tilde{\eta}}{\partial g_{n}}\right|^{2}\right)^{\frac{1}{2}}
\end{aligned} \\
\leq \Phi+\frac{1}{2} \mu_{1} \alpha^{2} \sqrt{N} .
\end{gathered}
$$

Here, $(f)$ is obtained by the second-order expansion of the function $y(x)=\int_{a}^{x} f(t) \mathrm{d} t$ and the fact that with uniform channel quantization we have $g_{n}=g_{1}+(n-1) \alpha$. Moreover, (30.II) comes from $g_{n}=g_{1}+(n-1) \alpha, \forall n$. The first inequality in (30.III) is based on triangle inequality. Also, (g) follows from (30.I) and defining $\mu_{1} \doteq \max _{x \geq 0}\left\{\frac{\mathrm{d} f_{G}(x)}{\mathrm{d} x}\right\}$ (see (2) and (3) for the properties of the family of exponential distributions).

For the asymptotic optimality of the uniform amplitude quantizer, the necessary condition is that the gradient (30.III) goes to zero when $N$ increases. We use this condition and (30.III) to derive (16) as follows. For the family of exponentially-distributed 
variables, the term $\Phi=f_{G}\left(g_{N}\right)\left|\alpha-\frac{\int_{g_{N}}^{\infty} f_{G}(t) \mathrm{d} t}{f_{G}\left(g_{N}\right)}\right|$ in (30.II) goes to zero exponentially if $g_{N} \rightarrow \infty$. This is because using L'Hopital's rule we have

$\Phi=f_{G}\left(g_{N}\right)\left|\alpha+f_{G}(g)\left(\frac{d f_{G}(g)}{d g}\right)^{-1}\right| \leq f_{G}\left(g_{N}\right)\left(|\alpha|+\left|\mu_{2}\right|\right)$

if $g_{N} \rightarrow \infty$ (see (3)). On the other hand, for $g_{N}=g_{1}+(N-$ 1) $\alpha \rightarrow \infty$ and vanishing gradient, we should have $\alpha \geq \mathcal{O}\left(N^{-1}\right)$ for the step size where $\mathcal{O}(X)$ denotes the order of the function $X$. Therefore, in the extreme case we have

$$
\alpha=N^{-\varepsilon(N)}, \lim _{N \rightarrow \infty} \varepsilon(N) \rightarrow 1, \varepsilon(N) \leq 1, \forall N
$$

which leads to $\frac{1}{2} \mu_{1} \alpha^{2} \sqrt{N}=\mathcal{O}\left(N^{-\frac{3}{2}}\right)$ and, from (30.III),

$$
\|\nabla\{\tilde{\eta}\}\| \leq \mathcal{O}\left(e^{-N}\right)+\mathcal{O}\left(N^{-\frac{3}{2}}\right)=\mathcal{O}\left(N^{-\frac{3}{2}}\right) \rightarrow 0
$$

when $N$ increases. Finally, using the step size (32), we have $g_{n}=g_{1}+(n-1) N^{-\varepsilon(N)}$ and $\frac{g_{n}}{g_{n+1}} \rightarrow 1$ for large $n$ 's. Moreover, while (32) gives the optimality condition of the step size, the codebook that achieves the optimal scaling is not unique, and different step sizes can be determined as long as (32) is satisfied (for more discussions and some examples of proper quantization step sizes with asymptotically high number of quantization regions, see [20, Section II.C] as well).

\section{REFERENCES}

[1] D. J. Love et al., "An overview of limited feedback in wireless communication systems," IEEE J. Sel. Areas Commun., vol. 26, no. 8, pp. 13411365, Oct. 2008

[2] B. Makki and T. Eriksson, "On hybrid ARQ and quantized CSI feedback schemes in quasi-static fading channels," IEEE Trans. Commun., vol. 60, no. 4, pp. 986-997, Apr. 2012.

[3] T. T. Kim and M. Skoglund, "On the expected rate of slowly fading channels with quantized side information," IEEE Trans. Commun., vol. 55, no. 4, pp. 820-829, Apr. 2007.

[4] T. Kim and M. Skoglund, "Diversity-multiplexing tradeoff in MIMO channels with partial CSIT," IEEE Trans. Inf. Theory, vol. 53, no. 8, pp. 2743-2759, Aug. 2007.

[5] B. Makki, J. Li, T. Eriksson, and T. Svensson, "Throughput analysis for multi-point joint transmission with quantized CSI feedback," in Proc. IEEE VTC Fall, Sep. 2012, pp. 1-5.

[6] Y. Isukapalli and B. D. Rao, "Finite rate feedback for spatially and temporally correlated MISO channels in the presence of estimation errors and feedback delay," in Proc. IEEE GLOBECOM, Nov. 2007, pp. 2791-2795.

[7] J. Zheng and B. D. Rao, "Analysis of multiple antenna systems with finite-rate channel information feedback over spatially correlated fading channels," IEEE Trans. Signal Process., vol. 55, no. 9, pp. 4612-4626, Sep. 2007.

[8] J. Zheng and B. D. Rao, "Capacity analysis of correlated multiple antenna systems with finite rate feedback," in Proc. IEEE ICC, Jun. 2006, vol. 9, pp. 4173-4179.

[9] W. Xu, C. Zhao, and Z. Ding, "Limited feedback design for MIMO broadcast channels with ARQ mechanism," IEEE Trans. Wireless Commun., vol. 8, no. 4, pp. 2132-2141, Apr. 2009.

[10] W. Xu, C. Zhao, and Z. Ding, "Optimisation of limited feedback design for heterogeneous users in multi-antenna downlinks," IET Commun., vol. 3 , no. 11, pp. 1724-1735, Nov. 2009.
[11] D. J. Love, R. W. Heath, and T. Strohmer, "Grassmannian beamforming for multiple-input multiple-output wireless systems," IEEE Trans. Inf. Theory, vol. 49, no. 10, pp. 2735-2747, Oct. 2003.

[12] S. Zhou, J. Gong, and Z. Niu, "Distributed adaptation of quantized feedback for downlink network MIMO systems," IEEE Trans. Wireless Commun., vol. 10, no. 1, pp. 61-67, Jan. 2011.

[13] N. Jindal, "MIMO broadcast channels with finite-rate feedback," IEEE Trans. Inf. Theory, vol. 52, no. 11, pp. 5045-5060, Nov. 2006.

[14] R. Bhagavatula and R. W. Heath, "Adaptive limited feedback for sum-rate maximizing beamforming in cooperative multicell systems," IEEE Trans. Signal Process., vol. 59, no. 2, pp. 800-811, Feb. 2011.

[15] J. Zhang, M. Kountouris, J. G. Andrews, and R. W. Heath, "Achievable throughput of multi-mode multiuser MIMO with imperfect CSI constraints," in Proc. IEEE ISIT, Jul. 2009, pp. 2659-2663.

[16] L. Li, M. Pesavento, and A. B. Gershman, "Downlink opportunistic scheduling with low-rate channel state feedback: Error rate analysis and optimization of the feedback parameters," IEEE Trans. Commun., vol. 58, no. 10, pp. 2871-2880, Oct. 2010.

[17] J. Jin, Q. Wang, C. Lin, H. Yang, and Y. Wang, "Coordinated multipoint transmission with limited feedback," in Proc. IEEE GLOBECOM, Dec. 2010, pp. 850-854.

[18] M. Kountouris, R. de Francisco, D. Gesbert, D. T. M. Slock, and T. Salzer, "Multiuser diversity-multiplexing tradeoff in MIMO broadcast channels with limited feedback," in Proc. Asilomar, Nov. 2006, pp. 364-368

[19] S. H. Moon, S. R. Lee, J. S. Kim, and I. Lee, "Channel quantization for block diagonalization with limited feedback in multiuser MIMO downlink channels," J. Commun. Netw., vol. 16, no. 1, pp. 1-9, Feb. 2014.

[20] B. Khoshnevis and W. Yu, "Bit allocation law for multiantenna channel feedback quantization: Single-user case," IEEE Trans. Signal Process., vol. 59, no. 5, pp. 2270-2283, May 2011.

[21] B. Khoshnevis and W. Yu, "Bit allocation laws for multiantenna channel feedback quantization: Multiuser case," IEEE Trans. Signal Process., vol. 60, no. 1, pp. 367-382, Jan. 2012.

[22] "System level comparison of best-M and DCT-based CQI compression schemes," Huawei, RAN1 Meeting 47bis, Sorrento, Italy, R1-070368, Jan. 15-19, 2007. [Online]. Available: http://www.3gpp.org/DynaReport/ TDocExMtg--R1-47b--26241.htm

[23] B. Makki and T. Eriksson, "Efficient channel quality feedback signaling using transform coding and bit allocation," in Proc. IEEE 71st VTC Spring, May 2010, pp. 1-5.

[24] C. Shen, T. Liu, and M. P. Fitz, "On the average rate performance of hybrid-ARQ in quasi-static fading channels," IEEE Trans. Commun., vol. 57, no. 11, pp. 3339-3352, Nov. 2009.

[25] B. Makki and T. Eriksson, "On the performance of MIMO-ARQ systems with channel state information at the receiver," IEEE Trans. Commun., vol. 62, no. 5, pp. 1588-1603, May 2014.

[26] C. Shen and M. P. Fitz, "Hybrid ARQ in multiple-antenna slow fading channels: Performance limits and optimal linear dispersion code design," IEEE Trans. Inf. Theory, vol. 57, no. 9, pp. 5863-5883, Sep. 2011.

[27] L. Szczecinski, C. Correa, and L. Ahumada, "Variable-rate transmission for incremental redundancy hybrid ARQ," in Proc. IEEE GLOBECOM, Dec. 2010, pp. 1-5.

[28] J. L. Devore, Probability and Statistics for Engineering and the Sciences, 8th ed. Pacific Grove, CA, USA: Duxbury, 2011.

[29] K. Sayood, Introduction to Data Compression, 3rd ed. San Mateo, CA, USA: Morgan Kaufmann, 2006.

[30] V. Prelov and S. Verdu, "Second-order asymptotics of mutual information," IEEE Trans. Inf. Theory, vol. 50, no. 8, pp. 1567-1580, Aug. 2004.

[31] L. Sboui, Z. Rezki, and M. Alouini, "Achievable rate of spectrum sharing cognitive radio systems over fading channels at low power regime," IEEE Trans. Wireless Commun., vol. 13, no. 11, pp. 6461-6473, Nov. 2014.

[32] X. Li, S. Jin, M. R. McKay, X. Gao, and K. K. Wong, "Capacity of MIMOMAC with transmit channel knowledge in the low SNR regime," IEEE Trans. Wireless Commun., vol. 9, no. 3, pp. 926-931, Mar. 2010.

[33] E. M. Stein and R. Shakarchi, Real Analysis: Measure Theory, Integration, Hilbert Spaces. Princeton, NJ, USA: Princeton Univ. Press, 2008.

[34] T. Agoh and K. Dilcher, "Reciprocity relations for Bernoulli numbers," Amer. Math. Monthly, vol. 115, no. 3, pp. 237-244, 2008

[35] T. Eriksson and E. Agrell, "Lattice-Based Quantization part II," Dept. Inf. Theory, Chalmers Univ. Technol., Goteborg, Sweden, Oct. 18, 1996, Tech. Rep.

[36] J. Mirza, P. A. Dmochowski, P. J. Smith, and M. Shafi, "Limited feedback multiuser MISO systems with differential codebooks in correlated channels," in Proc. IEEE ICC, Jun. 2013, pp. 5386-5391. 


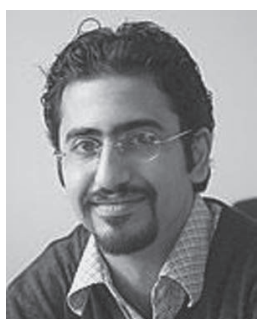

Behrooz Makki was born in Tehran, Iran. He received the B.Sc. degree in electrical engineering from Sharif University of Technology, Tehran, Iran, and the M.Sc. degree in bioelectric engineering from Amirkabir University of Technology, Tehran, Iran, respectively. He received the Ph.D. degree in communication engineering from Chalmers University of Technology, Gothenburg, Sweden. Since 2013, he has been working as a Postdoc at Chalmers University. His current research interests include partial channel state information (CSI) feedback, hybrid automatic repeat request, green communication, correlated fading channels, spectrum sharing, relay, satellite, and CoMP networks.

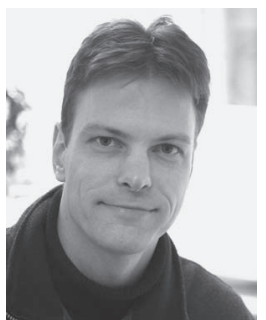

Tommy Svensson (S'98-M'03-SM'10) received the $\mathrm{Ph} . \mathrm{D}$. degree in information theory from Chalmers University of Technology in 2003, and he has worked at Ericsson $\mathrm{AB}$ with core networks, radio access networks, and microwave transmission products. He is Associate Professor in Communication Systems at Chalmers University, where he is leading the research on air interface and wireless backhaul networking technologies for future wireless systems. He was involved in the European collaborative research projects WINNER I, II, WINNER+, and ARTIST4G, which have contributed to the 3GPP LTE standards, and he is currently active in the EU FP7 METIS project targeting solutions for $5 \mathrm{G}$. His main research interests are in design and analysis of physical layer algorithms, multiple access, resource allocation, cooperative systems, moving relays/cells/networks, as well as satellite systems. He has co-authored two books and more than 100 journal and conference papers. He is chairman of the IEEE Sweden Vehicular Technology/Communications/Information Theory Chapter, and Coordinator of the Communication Engineering Master's Program at Chalmers.

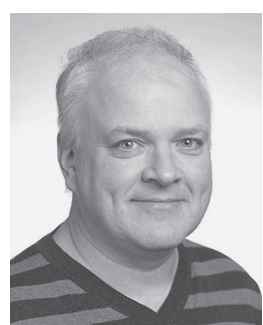

Thomas Eriksson received the Ph.D. degree in information theory from Chalmers University of Technology, Gothenburg, Sweden, in 1996. In 1997 and 1998, he was at AT\&T Labs Research in Murray Hill, NJ, USA, and in 1998 and 1999 he was at Ericsson Radio Systems AB, Kista, Sweden. Since 1999, he has been at Chalmers University, where he is a Professor in communication systems. Further, he was a Guest Professor at Yonsei University, $\mathrm{S}$. Korea, in 2003-2004. He is currently Vice Head of the Department of Signals and Systems at Chalmers, with responsibility for undergraduate and master education. His research interests include communication, data compression, and modeling and compensation of non-ideal hardware components (e.g., amplifiers, oscillators, modulators in communication transmitters and receivers).

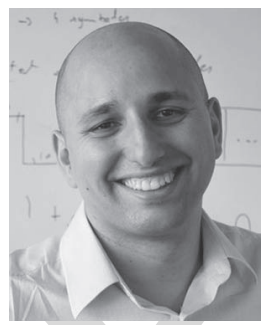

Mérouane Debbah (S'01-M'04-SM'08-F'15) received the M.Sc. and Ph.D. degrees from Ecole Normale Supérieure de Cachan, France, in 1996. He worked for Motorola Labs, Saclay, France, from 1999 to 2002, and the Vienna Research Center for Telecommunications (Vienna, Austria) until 2003. From 2003 to 2007, he joined the Mobile Communications Department of the Institut Eurecom (Sophia Antipolis, France) as an Assistant Professor. Since 2007, he has been a Full Professor at Supelec (Gifsur-Yvette, France). From 2007 to 2014, he was director of the Alcatel-Lucent Chair on Flexible Radio. Since 2014, he has been Vice-President of the Huawei France $\mathrm{R} * \mathrm{D}$ Center and Director of the Mathematical and Algorithmic Sciences Lab. His research interests are in information theory, signal processing, and wireless communications. He is an Associate Editor in Chief of the journal Random Matrix: Theory and Applications and was an Associate and Senior Area Editor for IEEE TRANSACTIONS ON SignAL PROCESSING respectively in 2011-2013 and 2013-2014. Dr. Debbah is a recipient of the ERC grant MORE (Advanced Mathematical Tools for Complex Network Engineering). He is a WWRF Fellow and a member of the academic senate of Paris-Saclay. He is the recipient of the Mario Boella award in 2005, the 2007 IEEE GLOBECOM Best Paper Award, the Wi-Opt 2009 Best Paper Award, the 2010 Newcom++ Best Paper Award, the WUN CogCom Best Paper 2012 and 2013 Award, the 2014 WCNC Best Paper Award as well as the Valuetools 2007, Valuetools 2008, CrownCom2009, Valuetools 2012 and SAM 2014 Best Student Paper Awards. In 2011, he received the IEEE Glavieux Prize Award and in 2012, the Qualcomm Innovation Prize Award. 